November 9, 2018 10:37 WSPC/Guidelines lecture

International Journal of Modern Physics B

(C) World Scientific Publishing Company

\title{
Weak quenched disorder and criticality: resummation of asymptotic(?) series
}

\author{
Yu. Holovatch \\ Institute for Condensed Matter Physics, National Academy of Sciences of Ukraine, \\ Lviv, UA-79011, Ukraine \\ Ivan Franko National University of Lviv, \\ Lviv, UA-79005, Ukraine \\ V. Blavats'ka and M. Dudka \\ Institute for Condensed Matter Physics, National Academy of Sciences of Ukraine, \\ Lviv, UA-79011, Ukraine \\ C. von Ferber \\ Physikalisches Institut, Theoretische Polymerphysik, Universität Freiburg, \\ D-79104 Freiburg, Germany \\ R. Folk \\ Institut für theoretische Physik, Johannes Kepler Universität Linz, \\ Linz, A-4040, Austria \\ T. Yavors'kii \\ Ivan Franko National University of Lviv, \\ Lviv, UA-79005, Ukraine \\ Received (received date) \\ Revised (revised date)
}

\begin{abstract}
In this review paper, we discuss the influence of weak quenched disorder on the critical behavior in condensed matter and give a brief review of available experimental and theoretical results as well as results of MC simulations of these phenomena. We concentrate on three cases: (i) uncorrelated random-site disorder, (ii) long-range-correlated random-site disorder, and (iii) random anisotropy.

Today, the standard analytical description of critical behavior is given by renormalization group results refined by resummation of the perturbation theory series. The convergence properties of the series are unknown for most disordered models. The main object of this review is to discuss the peculiarities of the application of resummation techniques to perturbation theory series of disordered models.
\end{abstract}

\section{Introduction}

There are two main questions we want to discuss in this paper. The first question is a physical one: how does disorder influence criticality? Taken that a non-disordered 
("ideal") system possesses a phase transition and a critical behavior governed by some scaling laws, will these universal laws be altered by disorder introduced into the system? The second question elaborated below is related to the unknown convergence properties of the series that result from the perturbative treatment of the corresponding models.

The first question we address here is rather specific since it concerns properties in a narrow region of the phase diagram in the vicinity of a critical point. Nonetheless, in order to give an extensive description of current studies in the field we are forced to restrict the subject further. In particular, in the present paper we will be interested only in static critical properties leaving the bulk of dynamic phenomena outside of our scope. We will choose three-dimensional systems of infinite size ignoring the influence of surfaces on the critical behaviour though such problems are very attractive 1 . Furthermore, we will address the subtle limit of weak disorder where the random system can be treated as perturbed with respect to an "ideal" one; this disregards the class of phenomena relevant in the strong disorder limit, like those appearing in the vicinity of a percolation threshold. Finally, we will touch only the quenched "frozen" disorder. To summarize, the main scope of our review will cover the static criticality of random quenched three-dimensional systems. In spite of our restriction of the subject, a lot of fascinating and still unsolved problems remain as we will try to convince the reader in our subsequent exposition.

To get a quantitative description of the phenomena occurring in the vicinity of the critical point it is standard to rely on renormalization group methods 2 . In this approach the physical quantities describing the critical behavior are obtained in the form of perturbation theory series. For some ideal (undiluted) systems these series have proven to be asymptotic and to have a zero radius of convergence. Therefore, special resummation methods are used to extract reliable data from the series. However, so far there is no proof of the asymptotic nature of the perturbation theory series for disordered (diluted) models. Moreover, there exist serious indications of possible non-asymptotic divergences. Nonetheless, the bulk of results that were obtained from the perturbation theory expansions of disordered models are presented as if they were asymptotic. Here arises the second question we address in this paper: how does the possible non-asymptotic nature show up in the analysis of perturbation theory expansions for the models with weak quenched disorder? Putting the first and the second questions together, the main goal of the current review will be to discuss what information about the influence of weak quenched disorder on the critical behavior can one extract working with renormalization group perturbation theory series.

The set-up of the paper is as follows. In the next Section 2 we discuss different models of weak quenched disorder and introduce the physical quantities used for their description. Here, of main interest for us will be systems with non-correlated point-like defects, systems with long-range correlated disorder and systems with disorder in the form of random anisotropy. In Section 3 we give examples of ex- 
perimental realizations of such systems. These include: site-diluted magnets, $\mathrm{He}^{4}$ in porous medium, magnets with extended impurities, polymers in random media, some amorphous rare-earth-transition metal alloys. The effective Hamiltonians for the disordered models under consideration are given in Section 4 together with the main relations of the field theoretical renormalization group theory. In Section 5 we describe the methods of resummation of divergent series and in Section 6 we display the results obtained by their application to the field theoretical renormalization group expansions. We conclude and give an outlook in the Section 7 .

\section{Different types of weak quenched disorder}

We start our consideration from one of the simplest and most carefully studied models which is introduced as a generalization of the hierarchy of the lattice Ising model, the $X Y$-model and the Heisenberg model. Consider a simple (hyper)cubic lattice of the dimension $d$, to each site of which it is prescribed a $m$-component vector $\vec{S}$ with a fixed length (for convenience of further notation one usually sets $|\vec{S}|=\sqrt{m}$ ). Imposing a pair interaction with the energy proportional to the scalar products between pairs of spins, one defines the Stanley model (it is also known as $m$-vector, $O(m)$-symmetric or generalized classical Heisenberg model). The Hamiltonian of the Stanley model reads $\mathrm{G}$ :

$$
H=-\frac{1}{2} \sum_{\mathbf{R}, \mathbf{R}^{\prime}} J\left(\left|\mathbf{R}-\mathbf{R}^{\prime}\right|\right) \vec{S}_{\mathbf{R}} \cdot \vec{S}_{\mathbf{R}^{\prime}}
$$

where the $d$-dimensional vectors $\mathbf{R}$ span the sites of a simple (hyper)cubic lattice (see Fig. 1) and $J\left(\left|\mathbf{R}-\mathbf{R}^{\prime}\right|\right)$ is an isotropic and translationally invariant short-range interaction. The Stanley model has further been generalized by analytic continuation in $m$ to describe critical properties of self-avoiding walks $(m=0)$, spherical ferromagnet $(m=\infty)$, the Gaussian model $(m=-2)$, etc.

A many-body system described by the model (if) can undergo a continuous phase transition into a ferromagnetic phase. Yet, the transition occurs only for lattice dimensions $d$ larger than a certain lower critical dimension $d_{\text {Low }}$. The numerical value of $d_{\text {Low }}$ is a function of the spin component number $m$. If the Hamiltonian (1) remains invariant under continuous rotations in the space of vectors $\vec{S}$, i.e. $m \geq 2$, one can show that $d_{\text {Low }}=2$. Otherwise, for $m=1$ (the Ising model) the continuous $O(m)$ symmetry degenerates into a discrete one corresponding to the invariance of the Hamiltonian(1i ) under discrete flip of all spins to the opposite direction. In this case $d_{\text {Low }}=1$.

In the theory of critical phenomena one is interested in the peculiarities of the behavior in the vicinity of the critical temperature $T_{c}>0$. The critical temperature essentially depends on microscopic details of the model. However, asymptotically close to $T_{c}$ the thermodynamic and correlation functions for very different systems may depend on the external parameters by the same power-like functions. These functions are primarily characterized by their critical exponents. 


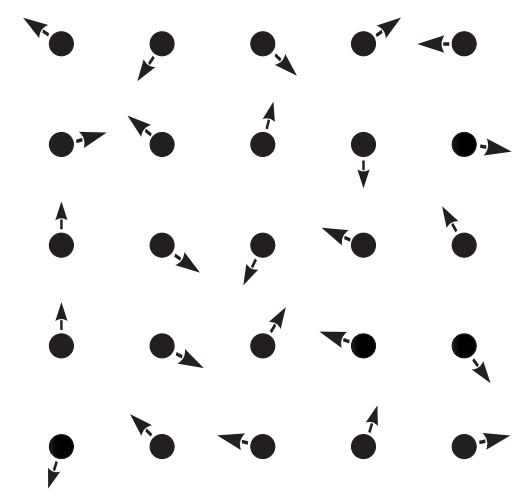

Fig. 1. The Stanley model describes a system of $m$-component vector "spins" occupying sites of a $d$-dimensional (hyper)cubic lattice and interacting by means of a short-range translationallyinvariant force.

The critical exponent $x$ of a physical observable $\mathcal{O}(\tau)$ asymptotically close to the critical point $T_{c}$ is defined by $5 \equiv \lim _{\tau \rightarrow 0} \frac{\ln \mathcal{O}(\tau)}{\ln |\tau|}$, where $\tau=\left(T-T_{c}\right) / T_{c}$ is the reduced distance to the critical point. For instance, the magnetic susceptibility $\chi$ diverges as:

$$
\chi \simeq \Gamma_{ \pm}|\tau|^{-\gamma}, \quad \tau \rightarrow 0
$$

where $\gamma$ is the susceptibility critical exponent, while $\Gamma_{+}$and $\Gamma_{-}$are critical amplitudes above and below the critical point respectively. The power law of type (2) is exact in the asymptotic regime $\tau \rightarrow 0$.

The definitions of the most common static critical exponents of a magnet are provided in Table 1.

Table 1. Critical exponents governing the power-like behavior of thermodynamic and correlation functions asymptotically close to $T_{c}$. Here, $M$ is the magnetization, $\chi$ is the magnetic susceptibility, $C_{H}$ is the heat capacity at constant external magnetic field $H ;\langle\delta M(0) \delta M(\mathbf{R})\rangle$ is the pair correlation function of the magnetization fluctuations and $\xi$ is the correlation length.

$$
\begin{array}{l|l}
M \propto(-\tau)^{\beta}\left(T \rightarrow T_{c}^{-}, H=0\right) & \langle\delta M(0) \delta M(\mathbf{R})\rangle \propto \exp (-|\mathbf{R}| / \xi)\left(T \neq T_{c}, H=0\right) \\
H \propto|M|^{\delta} \operatorname{sgn}(M)\left(T=T_{c}, H \neq 0\right) & \xi \propto \tau^{-\nu}\left(T \rightarrow T_{c}^{+}, H=0\right) \\
\chi \propto \tau^{-\gamma}\left(T \rightarrow T_{c}^{+}, H=0\right) & \langle\delta M(0) \delta M(\mathbf{R})\rangle \propto|\mathbf{R}|^{-(d-2+\eta)}\left(T=T_{c}, H=0\right) \\
C_{H} \propto \tau^{-\alpha}\left(T \rightarrow T_{c}^{+}, H=0\right) &
\end{array}
$$

In contrast to the critical temperature, the values of the critical exponents do not depend on microscopic details of the Hamiltonian and are determined only by the global properties of the model. For a short-range interaction the global properties are the lattice dimension $d$, the dimension $m$ and other symmetry properties of the order parameter. Due to the fact that the critical exponents may be identical 
for systems with very different microscopic nature, the critical exponents are called universal. Subsequently, if the critical properties of two systems are described by the same set of scaling functions and an identical set of critical exponents they are said to belong to the same universality class.

In the case of the $O(m)$-symmetric model (1D) the critical exponents are functions of the lattice and spin dimensions $d$ and $m$. However, if $d$ is greater than some upper critical dimension $d_{\mathrm{Up}}=4$, the critical exponents actually do not depend on $m$ and take mean field values $\gamma=1, \nu=1 / 2, \eta=0, \beta=1 / 2$. For $d_{\text {Low }} \leq d \leq d_{\mathrm{Up}}$ the critical exponents show non-trivial dependence on the spin dimension $m$ and need further consideration. In particular, for space dimension $d=3$ the critical exponents of the model (1) have been calculated so far with very high accuracy. The standard values of the critical exponents are listed in the Table 2.

Table 2. The stanfard numerical values of the critical exponents of the $d=3$ Stanley model (results of the Ref. 6). The cases $m=1,2,3$ correspond to Ising, $X Y$ - and Heisenberg models, $m=0$ reconstitutes the so-called polymer limit.

\begin{tabular}{|c|c|c|c|c|c|}
\hline$m$ & $\gamma$ & $\nu$ & $\eta$ & $\beta$ & $\alpha$ \\
\hline 0 & $1.1596 \pm 0.0020$ & $0.5882 \pm 0.0011$ & $0.0284 \pm 0.025$ & $0.3024 \pm 0.0008$ & $0.235 \pm 0.003$ \\
\hline 1 & $1.2396 \pm 0.0013$ & $0.6304 \pm 0.0013$ & $0.0335 \pm 0.025$ & $0.3258 \pm 0.0014$ & $0.109 \pm 0.004$ \\
\hline 2 & $1.3169 \pm 0.0020$ & $0.6703 \pm 0.0015$ & $0.0354 \pm 0.025$ & $0.3470 \pm 0.0016$ & $-0.011 \pm 0.004$ \\
\hline 3 & $1.3895 \pm 0.0050$ & $0.7073 \pm 0.0035$ & $0.0355 \pm 0.025$ & $0.3662 \pm 0.0025$ & $-0.122 \pm 0.010$ \\
\hline
\end{tabular}

The model (11) corresponds at criticality to a wide range of real systems, not always magnetic, and it is a convenient starting point to study critical phenomena. However, it suffers from obvious restrictions. Typical for real substances are properties which can be considered as deviations from the "ideal" structure of the Hamiltonian (1). Various types of defects spoil the strict translational invariance of the inter-spin interaction $J$ and/or can be effectively represented by additional terms in the Hamiltonian. In both cases, disorder introduces stochastic (random) variables in the Hamiltonian of the system. In the following, we will treat randomness introduced by either site dilution or random anisotropy.

The presence of non-magnetic impurities can be modeled by a class of sitediluted models $\mathrm{U}$. A site-diluted Stanley model is introduced by the Hamiltonian:

$$
H=-\frac{1}{2} \sum_{\mathbf{R}, \mathbf{R}^{\prime}} J\left(\left|\mathbf{R}-\mathbf{R}^{\prime}\right|\right) \vec{S}_{\mathbf{R}} \cdot \vec{S}_{\mathbf{R}^{\prime}} c_{\mathbf{R}} c_{\mathbf{R}^{\prime}}
$$

where $c_{\mathbf{R}}$ is an occupation number: it equals 1 when the site is occupied by a magnetic atom and 0 otherwise (see Fig. 2). Geometrically, the vacancies $c_{R}=0$ can be distributed independently according to the probability density

$$
P\left(c_{R}\right)=(1-p) \delta\left(c_{R}\right)+p \delta\left(1-c_{R}\right)
$$

with $p$ being the concentration of magnetic atoms. In the absence of correlations between the vacancies the ordered phase of the model (3) persists with a decrease of concentration $p$ up to some value $p_{c}$. The latter depends on the lattice type and 


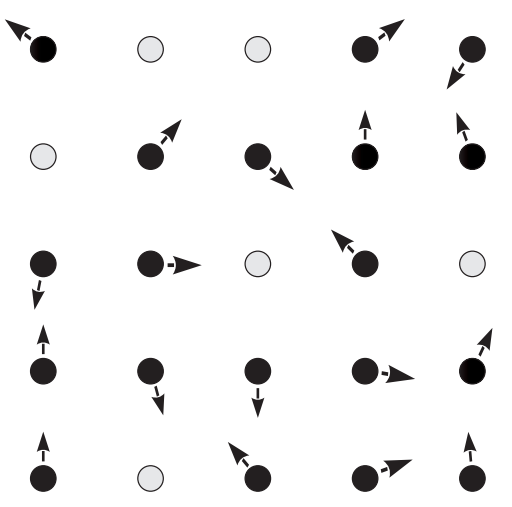

Fig. 2. The weakly diluted Stanley model is characterized by a small amount of randomly distributed (quenched) non-magnetic atoms which spoil the translationally-invariant structure of the spins in Fig. 1.

dimension and is equal to the percolation threshold $p_{\text {perc }}$ 8. Here, we are not interested in phenomena occurring in the vicinity of the percolation threshold $p \sim p_{\text {perc }}$ but rather we address the limit of weak dilution $p \simeq 1$. Since in the critical region impurities cause, in particular, a shift of the critical temperature, this property can be taken as a genuine property of disorder due to non-magnetic defects. In turn, the scale of the correlation of non-magnetic impurities becomes apparent by the fluctuations of the local temperature of the magnetic phase transition. In what follows below we will not consider frustrations in the exchange interaction $J(R)$ in (3) which may lead to the spin-glass phase as far as the main problem of interest for us is the second order ferromagnetic phase transition.

From the viewpoint of dynamics one may distinguish two main types of disorder. If the dynamics of the impurities has a characteristic time that is comparable to the relaxation times in the pure system, the impurity variables are treated identically to the "pure" dynamical variables and are a part of the disordered system phase space. The corresponding annealed disorder has been subject to numerous investigations and reveals trivial results at criticality. The so called Fisher renormalization 10 states that when a heat capacity critical exponent of an undiluted system $\alpha_{\text {pure }}$ is positive, then the critical exponent $\alpha$ of the corresponding annealed diluted system changes to $\alpha=\frac{-\alpha_{\text {pure }}}{1-\alpha_{\text {pure }}}$ and any other critical exponent $x$ of the annealed system is determined by the corresponding pure one $\left(x_{\text {pure }}\right)$ by a simple normalization of the form: $x=\frac{x_{\text {pure }}}{1-\alpha_{\text {pure }}}$. This explains the prevailing interest in systems with quenched disorder where the impurities can be considered as fixed. To obtain the thermodynamics of the quenched system one needs to perform the configurational average over an ensemble of disordered systems with different realization of the disorder.

The influence of uncorrelated weak disorder of type (4) on the critical behavior of the model (3) can be predicted by the so-called Harris criterion 12 . It states that 
only if the heat capacity critical exponent $\alpha_{\text {pure }}$ of the pure (undiluted) system is positive, i.e. the heat capacity diverges at the critical point, quenched disorder causes changes of the critical exponents. From Table I one concludes that only the Ising model is affected by short-range-correlated quenched disorder at criticality 12. Moreover, the heat-capacity critical exponent of the weakly-diluted Ising model should be negative as is suggested by the inequality $\nu \geq 2 / d$ proven for diluted systems 13 .

To explain possible generalizations of the model (3) with site occupation distribution (1) let us note, that (as it will be seen from Section 5) for well behaved distributions the two first moments as far as they exist determine the critical behaviour of the model. For the distribution (画) one gets:

$$
\begin{gathered}
\left\langle c_{\mathbf{R}}\right\rangle=p, \\
g\left(\left|\mathbf{R}-\mathbf{R}^{\prime}\right|\right)=\left\langle c_{\mathbf{R}} c_{\mathbf{R}^{\prime}}\right\rangle-\left\langle c_{\mathbf{R}}\right\rangle^{2}=p(1-p) \delta_{\mathbf{R}-\mathbf{R}^{\prime}},
\end{gathered}
$$

where $\langle\ldots\rangle$ means averaging with the distribution function (4), and $\delta_{\mathbf{R}-\mathbf{R}^{\prime}}$ is Kronecker's delta.

For the pair correlation function (6) one may consider 15 more general cases. In the first of the two generalizations treated in this review the quenched random impurities are strongly correlated in $\varepsilon_{d}$ dimensions and randomly distributed over the remaining $d-\varepsilon_{d}$ dimensions while in a second generalization we will treat long range correlations as described below. In the first case, the impurities are envisaged as parallel $\varepsilon_{d}$-dimensional objects, each extending along the coordinate directions symbolized as $\mathbf{R}_{\|}$, whereas in remaining $d-\varepsilon_{d}$ dimensions symbolized as $\mathbf{R}_{\perp}$ they are randomly distributed. The pair correlation function in this case is defined to yield 15 :

$$
g\left(\left|\mathbf{R}-\mathbf{R}^{\prime}\right|\right)=v_{0} \delta^{d-\varepsilon_{d}}\left(\mathbf{R}_{\perp}-\mathbf{R}_{\perp}^{\prime}\right), \quad \text { with } v_{0}>0 .
$$

The case of point-like uncorrelated impurities (and the pair correlation function (6)) is reconstituted from (7) by $\varepsilon_{d}=0$. Systems with impurities distribution governed by the pair correlation function (8) are not covered by the aforementioned Harris criterion. To show this, let us note that due to fluctuations in concentration appears the local transition temperature will vary. Denoting the deviation from the local transition temperature by $\tau(R)$ one can determine the mean deviation $\tau_{\xi^{d}}$ to the transition temperature in a region of correlated spins, i.e. in a volume $\xi^{d}$ with correlation length $\xi$, as the average of $\tau(\mathbf{R})$ over this region,

$$
\tau_{\xi^{d}}=\frac{1}{\xi^{d}} \int_{\xi^{d}} \mathrm{~d}^{d} R \tau(\mathbf{R})
$$

For convenience, we pass to the continuum space $R$ with subsequent change of Kronecker's deltas to $\delta$-functions and of sums to integrals.

The mean values (8) vary from one correlated volume $\xi$ to another. One can only speak consistently of a phase transition in the whole sample at a precise transition temperature if these variations become negligibly small as the transition is 
approached. The variation of the mean values $\tau_{\xi^{d}}$ in different correlated regions is characterized by their variance

$$
\Delta^{2}=\frac{1}{\xi^{2 d}} \int_{\xi^{d}} \mathrm{~d}^{d} R \int_{\xi^{d}} \mathrm{~d}^{d} R^{\prime} g\left(\left|\mathbf{R}-\mathbf{R}^{\prime}\right|\right) .
$$

For the weak impurity concentrations the shift in the local transition temperature is proportional to the concentration. This allows one to substitute the pair correlation function in (9) by the impurity pair correlation function (7). Evaluating (9) and using $\xi \sim \tau^{-\nu_{p}}$ (with $\nu_{p}$ being the correlation length critical exponent of the pure model) one finds that for $\tau \rightarrow 0$ :

$$
\Delta^{2} / \tau^{2} \sim \tau^{\left(d-\varepsilon_{d}\right) \nu_{p}-2} .
$$

This tends to zero, i.e. the variance becomes negligible, for $\tau \rightarrow 0$ if the exponent of $\tau$ is positive; if it is negative, however, the variance $\Delta$ goes to zero more slowly than $\tau$ itself, and the mean value $\tau$ becomes meaningless 16 . This is the indication, that the defects qualitatively change the transition, and it leads to the generalized Harris criterion for the relevance of extended defects on the critical behaviour:

$$
\varepsilon_{d}>d-\frac{2}{\nu_{p}} .
$$

Putting in (11) $\varepsilon_{d}=0$ one recovers the Harris criterion for the point-like weak quenched disorder 11 .

The second generalized correlation function that we treat here describes a powerlaw decrease of the correlations at large distances 4 :

$$
g\left(\left|\mathbf{R}-\mathbf{R}^{\prime}\right|\right) \sim\left|\mathbf{R}-\mathbf{R}^{\prime}\right|^{-a} .
$$

In what follows, defects which are correlated according to (12) with $a \geq d$ will be called short-range correlated, and correspondingly, with $a<d$ - long-rangecorrelated. The correlation function (12) has a direct physical interpretation: straight lines of impurities of random orientation are described by the case $a=d-1$, whereas randomly oriented planes of impurities correspond to $a=d-2$ 느. Moreover, different non-integer values of $a$ may be interpreted as randomly distributed impurities of fractal nature 17 . Weinrib and Halperin 4 showed that in the presence of longrange power-law correlations in the disorder the Harris criterion is also modified: for $a<d$ the disorder is relevant, if the correlation length critical exponent of the pure system obeys $\nu<2 / a$, while for $a \geq d$ the usual Harris criterion for the influence of short-range-correlated point defects applies. The derivation is similar to that presented above for the case of extended impurities.

As mentioned above, apart from site dilution as a second example of randomness we treat a random anisotropy present at all sites. Let us introduce a model for quenched weak random anisotropy proposed by Harris, Plischke, and Zuckermann 18. This model describes a regular lattice of magnetic ions, each of them being subject to a local anisotropy field of random orientation in the form of an easy axis. The magnitude of this field is the same for all sites. The Hamiltonian of the 
random anisotropy model (RAM) then has the form of the Hamiltonian (1) with an additional anisotropy term:

$$
\mathcal{H}=-\frac{1}{2} \sum_{\mathbf{R}, \mathbf{R}^{\prime}} J\left(\left|\mathbf{R}-\mathbf{R}^{\prime}\right|\right) \vec{S}_{\mathbf{R}} \cdot \vec{S}_{\mathbf{R}^{\prime}}-D \sum_{\mathbf{R}}\left(\hat{z}_{\mathbf{R}} \vec{S}_{\mathbf{R}}\right)^{2}
$$

Here, $D>0$ is an anisotropy strength (the case of $D<0$ corresponds to the presence of easy planes in the model), and $\hat{z}_{\mathbf{R}}$ is the unit vector pointing in the local (quenched) random direction of the uniaxial anisotropy.

Strong disorder at large ratio $D / J$, should destroy the second order phase transition. For small $D / J$ this is not so obvious. In addition to the global variables of a regular magnet (i.e. lattice dimension, type of interaction and spin symmetry) the low-temperature ordering in RAM is influenced also by the distribution of the random variables $\hat{z} \equiv \hat{z}_{\mathbf{R}}$ in (13). For non-correlated $\hat{z}$ the low-temperature ordering depends on the probability distribution $p(\hat{z})$ of the direction of anisotropy on a single site. As introduced by Aharony 19 two kinds of distribution functions that are relatively simple to study and that correspond to real physical situations, are generally considered. The first one corresponds to the isotropic case, when the random vector $\hat{z}$ points with equal probability in any direction of the $m$-dimensional hyperspace

$$
p(\hat{z}) \equiv\left(\int d^{m} \hat{z}\right)^{-1}=\frac{\Gamma(m / 2)}{2 \pi^{m / 2}},
$$

with $\Gamma(x)$ being Euler's gamma-function. The second one corresponds to the socalled cubic anisotropy, when the vector $\hat{z}$ points to any of the $2 m$ directions $\hat{k}_{i}$ along the edges of the $m$-dimensional hypercube:

$$
p(\hat{z})=\frac{1}{2 m} \sum_{i=1}^{m}\left[\delta^{(m)}\left(\hat{z}-\hat{k}_{i}\right)+\delta^{(m)}\left(\hat{z}+\hat{k}_{i}\right)\right] .
$$

The rationale for such a choice is to mimic the situation when an amorphous magnet still "remembers" the initial (cubic) lattice structure. Other distributions may be considered as well.

For the isotropic case (14) arguments similar to those of Imry and Ma 20 applied to the random-field Ising model demonstrate the absence of magnetization below $d=4$. Applying domain arguments 21 one supposes that the ground state will break into finite clusters of linear size $L$. This is possible if the bulk energy $\sim D L^{-d / 2}$ gained is larger than the domain wall energy. For isotropic spin systems the latter energy is $\sim J L^{d-2}$. This leads to the conclusion that below four dimensions a break up into domains is favored with a characteristic domain size $L_{0} \sim(J / D)^{2 /(4-d)}$. Other arguments were applied by Pelcovits et al. 22 . Assuming a ferromagnetic state they calculated the correlation function of transverse magnetization and obtained its divergence for $d<4$. However these arguments do not consider the anisotropic distributions $p(\hat{z})$. 
In the next section we introduce various physical systems described by disordered quenched models (3), (13). A brief review of available experimental and MC data for these systems is given as well.

\section{Realizations of weak quenched disorder: experiments and Monte-Carlo simulations}

In this section, we review critical phenomena in substances and their computer simulation that can be considered as realizations of the models discussed above. We start from weakly site-diluted quenched Stanley models. Among these, the bulk of results is known for the weakly diluted quenched Ising model as is suggested by the Harris criterion.

\subsection{Site-diluted uniaxial antiferromagnets}

Typical experimental realizations of the weakly diluted quenched Ising model are given by crystalline mixtures of two compounds. The first is an "Ising-like" anisotropic uniaxial antiferromagnet with dominating short-range interaction (e.g. $\left.\mathrm{FeF}_{2}, \mathrm{MnF}_{2}\right)$, the second one is non-magnetic $\left(\mathrm{ZnF}_{2}\right)$. Mixed crystals $\left(\mathrm{Fe}_{\mathrm{p}} \mathrm{Zn}_{1-\mathrm{p}} \mathrm{F}_{2}\right.$, $\mathrm{Mn}_{\mathrm{p}} \mathrm{Zn}_{1-\mathrm{p}} \mathrm{F}_{2}$ ) can be grown with high crystalline quality and very small concentration gradients providing an excellent realization of random substitutional disorder of magnetic ions $\left(\mathrm{Fe}^{+2}, \mathrm{Mn}^{+2}\right)$ by non-magnetic ones $\left(\mathrm{Zn}^{+2}\right)$.

Already the first experimental studies gave evidence of new critical behavior caused by a weak quenched dilution. In nuclear magnetic resonance measurements of the magnetization in $\mathrm{Mn}_{0.864} \mathrm{Zn}_{0.136} \mathrm{~F}_{2} 23$ the value of the magnetization exponent $\beta=0.349 \pm 0.008$ was found to differ strongly from that in an undiluted sample. Within a few years this result was corroborated by nuclear scattering measurements of the magnetic susceptibility and correlation length critical exponents in $\mathrm{Fe}_{\mathrm{p}} \mathrm{Zn}_{1-\mathrm{p}} \mathrm{F}_{2} 2425$ and $\mathrm{Mn}_{\mathrm{p}} \mathrm{Zn}_{1-\mathrm{p}} \mathrm{F}_{2} 26$ at different dilutions. In particular, the latter study showed that critical behaviour of the susceptibility in $\mathrm{Mn}_{0.75} \mathrm{Zn}_{0.25} \mathrm{~F}_{2}$ 26 is governed by a critical exponent $\gamma$ which, consistently with the Harris criterion, differs from that of the "ideal" magnet (see Fig. 3). The linear birefringence measurements revealed the cusp-like behavior of the specific heat at the transition point with an exponent $\alpha=-0.09 \pm 0.0324$. This experimentally proved that within the error bars the hyperscaling relation $d \nu+\alpha=2$ is satisfied.

Crossover phenomena in diluted systems are governed in addition to the temperature by the concentration of the magnetic component. The experimentally obtained exponents are often reported to be effective ones (i.e. temperature- and dilution-dependent). However, already in the first experiments on the critical behavior of quenched diluted Ising-like antiferromagnets it appeared possible to reach the asymptotic region. Thus, studying the critical regime in Ref. 24 the authors found neither a region in reduced temperature $\tau$ where one finds "pure" Ising exponents nor any evidence of crossover from pure to random exponents. This was explained by the crossover either taking place outside the critical region or being 


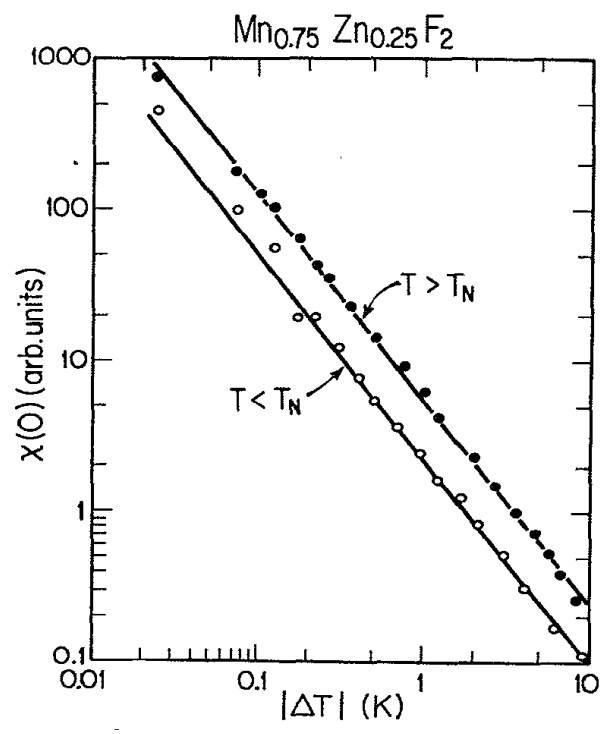

Fig. 3. Neutron scattering measurements of the susceptibility $\chi(0)$ in $\mathrm{Mn}_{0.75} \mathrm{Zn}_{0.25} \mathrm{~F}_{2} 26$. The solid lines are the results of fits to single power laws with exponents $\gamma \simeq 1.364$ above and below Néel temperature $T_{N}$. The critical behaviour is governed by a simple power law in the reduced temperature interval $4 \cdot 10^{-4}<|\tau|<2 \cdot 10^{-1}$.

too slow. In Ref. 27 the crossover from pure to diluted critical behavior was studied and a magnetization exponent $\beta=0.36 \pm 0.01$ was found that does not change under dilution for $p \leq 0.05$. The crossover occurs within a very narrow range of $\tau$ at relatively large values of $\tau$. In Ref. 26 excellent agreement of the measured exponents $\gamma=1.364 \pm 0.076$ and $\nu=0.715 \pm 0.035$ with the theoretical values of the diluted Ising model was obtained for the temperature range $4 \cdot 10^{-4} \leq \tau \leq 10^{-1}$ and $p=0.75$. The valye of the critical exponent $\beta$ was also the subject of a crossover analysis in Refs. 22.29. Ref. 28 concludes that the experimental errors are too large in order to distinguish between the pure Ising model and the diluted Ising model critical behavior. In Ref. 29 no crossover was found after the correction-to-scaling had been taken into account, and the diluted Ising model critical behavior was found in the whole temperature range.

It is known that the diluted Ising magnet in a uniform magnetic field $H$ along the uniaxial direction exhibits static critical behavior of the random-field Ising model 30. Such experiments give additional information about the critical behavior of the diluted Ising model when performed for $H=0$ 31. A comprehensive account of the experimentally measured values of the critical exponents of diluted uniaxial magnets is given in a recent review 32 . 


\subsection{Disordered Heisenberg-like magnets}

Another experimental realization of the weakly diluted Stanley model are given by magnetic systems described by the site-disordered Heisenberg model Some amorphous magnets as $\mathrm{Fe}_{90+\mathrm{x}} \mathrm{Zr}_{10-\mathrm{x}}, \mathrm{Fe}_{90} \mathrm{M}_{\mathrm{y}} \mathrm{Zr}_{10}(\mathrm{M}=\mathrm{Co}, \mathrm{Mn}, \mathrm{Ni}) 33.34$ and transition-metal based magnetic glasses 35.36 as well as disordered crystalline materials as $\mathrm{Fe}_{100-\mathrm{x}} \mathrm{Pt}_{\mathrm{x}}$ 37, $\mathrm{Fe}_{70} \mathrm{Ni}_{30} 36$ and Eu-chalcogenide solid solution systems $\left(\mathrm{Eu}_{\mathrm{x}} \mathrm{Sr}_{1-\mathrm{x}} \mathrm{S}_{0.5} \mathrm{Se}_{0.5}, \mathrm{Eu}_{\mathrm{x}} \mathrm{Sr}_{1-\mathrm{x}} \mathrm{S}, \mathrm{Eu}_{\mathrm{x}} \mathrm{Ba}_{1-\mathrm{x}} \mathrm{S}, \mathrm{Eu}_{\mathrm{x}} \mathrm{La}_{1-\mathrm{x}} \mathrm{S}\right)^{?, ?, ?, ?}$ belong to this group of systems.

According to the Harris criterion the critical behaviour of the diluted Heisenberg model should remain unaltered in its asymptotics with critical exponents of the $d=3$ pure Heisenberg model (see the last line of Table 2). However, the experiments give a wide scattering of the values of the critical exponents. Often the reason is that the asymptotic region still is not reached and only the effective exponent is observed. In particular, the value of the magnetic susceptibility effective critical exponent $\gamma_{\text {eff }}$ with decrease of distance to the critical point goes through a peak to its asymptotic value which coincides with magnetic susceptibility critical exponent of pure Heisenberg magnets. The dependence of $\gamma_{e f f}$ on reduced temperature $\tau$ is presented in Fig. 1 taken from the recent paper 34. The effective behaviour also was studied by Monte Carlo simulations 39 , the results show that $\gamma_{\text {eff }}$ has a maximum while approaching the asymptotic regime. Such form of temperature dependence of effective susceptibility critical exponent is corroborated by recent theoretical investigations 40

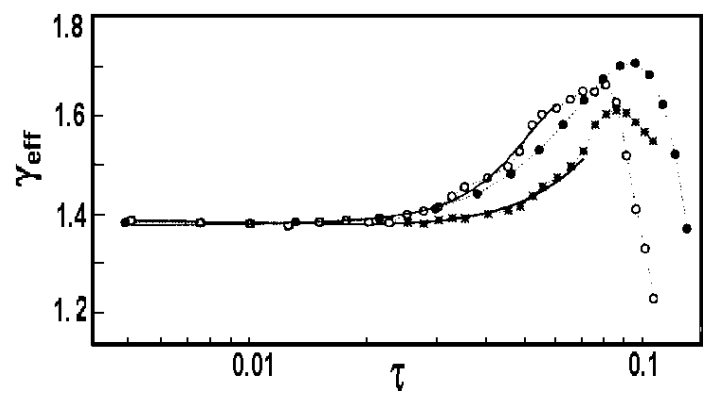

Fig. 4. Experimentally measured isothermal susceptibility effective critical exponent $\gamma_{e f f}$ for Fe90-x $\mathrm{Mn}_{\mathrm{x}} \mathrm{Zr}_{10}$ for different values of $x$ 制 $\left(\tau=\left(T-T_{c}\right) / T_{c}\right)$.

\section{3. $\mathrm{He}^{4}$ in a porous medium}

The listed experimental evidences of the critical behavior of the weakly diluted quenched Ising model confirm the Harris criterion. However, the bulk of the data was obtained for the magnetic phase transition. Due to the universal character of the critical exponents the model (1) can describe universal properties of critical 
phenomena of completely different microscopic nature. An example is the transition of liquid helium- 4 to the superfluid state which belongs to the Stanley model universality class at $m=2$. The uncorrelated point-like disorder (4) in the case of a fluid can be introduced by a porous medium 41 . Recently, high precision experiments on the critical behavior of liquid helium-4 near the superfluid transition in a porous medium 42 confirmed the irrelevance of quenched disorder, again in correspondence with the Harris criterion since the specific heat exponent near the superfluid transition is slightly negative 43 . More data about effect of disorder on the $\lambda$-transition of $\mathrm{He}^{4}$ may be found in recent review 44 .

\subsection{MC studies of random Ising model}

Another way to study the critical behavior of the model (3) is to use computer Monte-Carlo experiments. However, in contrast to experiments, the first studies of critical behaviour of the weakly diluted Ising model on a simple cubic lattice 45 revealed critical exponents that were identical within the numerical error with the corresponding exponents of the pure (undiluted) system within a wide dilution region. Later, these data were objected by subsequent $\mathrm{MC}$ simulations on larger lattices 46 , that obtained critical exponents varying continuously with the magnetic sites concentration $p$. Indications of a change of the order parameter critical exponent $\beta$ upon dilution initiated an extension of the studies to determine the other critical exponents and to check the scaling for disordered systems. Finally, the Swedsen-Wang algorithm that avoids the critical slowing down of the relaxation was applied to the $d=3$ diluted Ising model 47 and resulted in critical exponents for the susceptibility and correlation length that were independent of concentration over a wide range gf dilution.

Due to Refs. 47.48 and especially 49.50 it became clear that the concentration dependent critical exponents found in previous MC simulations are effective ones, characterizing the approach to the asymptotic region. The effective exponents $\gamma, \beta$ and $\zeta=1-\beta$ (the last one describes the divergence of the magnetization-energy correlation function) were shown 49 to be concentration dependent in the concentration region $0.5 \leq p<1$. These data were refined three years later 50 resulting in more accurate estimates for the above mentioned exponents and the critical exponent $\nu$ of the correlation length with continuously varying values.

The critical behavior of the $d=3$ diluted Ising model was reexamined recently in Ref. 51. The study was based on the crucial observation that it is important to take into account the leading correction-to-scaling term in the infinite volume extrapolation of the MC data. The simulations confirmed the universality of the critical exponents of the $d=3$ diluted Ising model over a wide region of concentrations (see Fig. F) . In particular the value of the correction-to-scaling exponent $\omega$ was found to be $\omega=0.37 \pm 0.06$ which is almost half as large as the corresponding value in the pure $d=3$ Ising model $\omega=0.799 \pm 0.011$ 目. The smallness of $\omega$ in the dilute case explains its importance for an analysis of the asymptotic critical behavior. 
The other exponents were determined as: $\beta=0.3546 \pm 0.0028, \gamma=1.342 \pm 0.010$, $\nu=0.6837 \pm 0.0053$. We refer the interested reader to the papers 5232 where the numerical values of the exponents obtained in the MC simulations are listed.

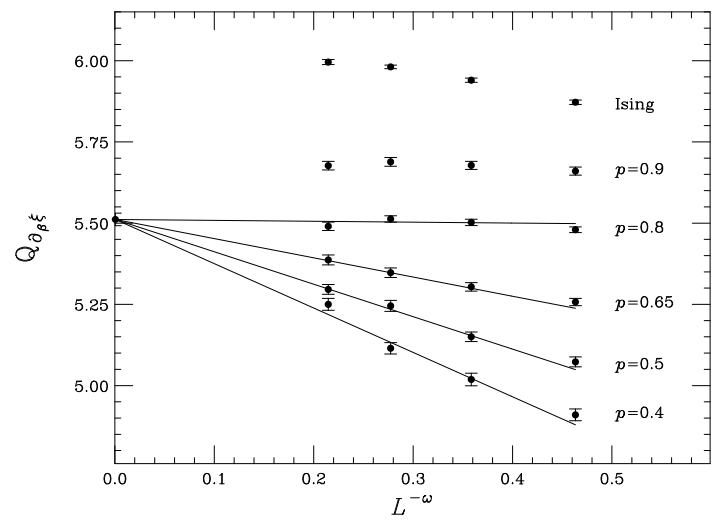

Fig. 5. Determination of the correction-to-scaling exponent $\omega$ in the MC simulations of Ref. 51 . Quantity $Q_{\partial_{\beta} \xi}=2^{1+1 / \nu}$ is plotted for different dilutions $0.4 \leq p \leq 1$ and lattice sizes $8 \leq L \leq 128$. One can see that at $p=0.9$ the system is crossing over from the pure Ising fixed point to the diluted one even for $L=128$. The solid lines correspond to a fit $\omega=0.37$ yielding the same infinite volume extrapolation for all $p \leq 0.8$.

\subsection{Magnets with long-range-correlated quenched disorder}

Whereas at present many experimental and MC simulation data are available for magnetic systems with random-site uncorrelated disorder, the critical behavior of systems with long-range-correlated disorder is much less investigated. Therefore, in this subsection that is devoted to systems with this type of disorder we present only a few works where its influence has been studied.

In magnets, long-range-correlated disorder may be present in the form of continuously distributed dislocations and disclinations, so-called extended structural defects. These defects may have the form of lines or planes of random orientation 53 or may form some sponge-like fractal objects, which are considered as aggregation clusters 17. The particular case of systems with dislocation lines or planes of parallel orientation, is investigated in Ref. 4

Recently, Ballesteros and Parisi 55 presented Monte-Carlo simulations of the diluted Ising model in three dimensions with extended defects in the form of lines of parallel orientation. Finite-size scaling techniques were used to compute the critical exponents of these systems, taking into account the strong scaling corrections. The obtained value for the exponent $\nu$ is compatible with the analytic predictions 14 . In Ref. 56 the three-dimensional so-called thermally diluted Ising systems were studied in Monte-Carlo simulations. Here, at the characteristic ordering temperature $\theta$, the 
spins of the dominant type (concentration $p \geq 0.5$ ) are taken as the location of the magnetic atoms, and the rest are taken as magnetic vacancies. The structure of the realization is fixed thereafter for all temperatures at which the magnetic interactions are subsequently investigated. If $\theta$ happens to coincide with the critical temperature of the undiluted Ising model, the vacancies are in randomly located points, but with a long-range-correlated distribution. It was found that the simulated critical exponents of thermally diluted Ising systems at criticality agree fairly well with theoretical predictions of Weinrib and Halperin 14.

We adduce here also the interesting result of Monte-Carlo study of three-dimensional Ising model with bond disorder, which is random in the vertical direction and correlated in $\varepsilon_{d}=2$ horizontal directions 57 . The results demonstrate a sharp second-order phase transition, with exponents of new universality class. However, it seems not to be of the same universality class as the model of extended defects presented by Dorogovtsev 15.

\subsection{Polymers in random media}

Another example of critical behavior in the presence of weak quenched disorder is found for the scaling properties of long flexible polymer chains in random media. The question of how linear polymers behave in disordered media is not only interesting from a theoretical point of view, but is also relevant for understanding transport properties of polymer chains in porous media, such as enhanced oil recovery, gel electrophoresis, gel permeation chromatography etc.

It is established that the universal scaling properties of long flexible polymer chains in a good solvent are perfectly described within a model of self-avoiding walks (SAWs) on a regular lattice 58. Their scaling properties in the limit of an infinite number of steps are governed by the same scaling laws as the pure (undiluted) Stanley model at the critical point in the formal $m \rightarrow 0$ limit 59 . In particular, for the average square end-to-end distance $\left\langle R^{2}\right\rangle$ and the number of configurations $Z_{N}$ of a SAW with $N$ steps on a regular lattice one finds in the asymptotic limit $N \rightarrow \infty$ :

$$
\left\langle R^{2}\right\rangle \sim N^{2 \nu}, \quad Z_{N} \sim \tilde{z}^{N} N^{\gamma-1}
$$

where $\nu$ and $\gamma$ are the universal correlation length and susceptibility exponents for the $m=0$ model that only depend on the space dimensionality $d$ and $\tilde{z}$ is a non-universal fugacity. For $d=3$ the exponents are given in Table 1 .

The problem of SAWs on randomly diluted lattices, which may serve as a model of polvmer chains in a porous medium, has been the subject of intensive discussion 60.61 62.63.64.65.60.67. The main question of interest here is: does a small amount of quenched structural point-like defects in the medium induce changes to the universal properties of the polymer macromolecules? One should be careful in applying the Harris criterion "naively" to the SAW problem. Although the critical exponent $\alpha$ of a SAW on the $d=3$ dimensional pure lattice is positive $6(\alpha(d=3)=0.235 \pm 0.003)$, 
a weak quenched short-range-correlated disorder does ngt alter the SAW critical exponents. This statement has been proven by Harris 61 and confirmed later by renormalization group results 62 . Numerical results for these systems available from Monte-Carlo simulations also lead to the conclusion that such disorder does not affects the scaling behavior of SAWs 6568 .

On the other hand, universal properties of SAWs on a percolation cluster obey new scaling laws (see, e.g. 63.69 and references therein).

Another interesting problem is the scaling behavior of polymers in disordered media whep the defects are correlated or belong to some sponge-like structure. In Refs. 6970 it was found that the asymptotic behavior of SAWs in long-rangecorrelated disorder of type (12) is governed by a set of critical exponents which are different from that in the pure case. This recent result waits for its experimental verification.

\subsection{Random anisotropy magnets}

Among disordered materials containing magnetic elements there are some intermetallic amorphous alloys, which are characterized not by a random-site disorder, but by a disorder in the form of a random anisotropy. Random anisotropy is the most important characteristic of these metals and that results from their random closepacking structure. Typical examples of random anisotropic materials are amorphous rare-earth-transition metal alloys 18 . Their features are taken into account by the random anisotropy model (RAM) in (13). The model may be extended also to describe other intermetallic amorphous alloys containing rare-earth elements with asymmetric charge distribution 11 .

The original example for the RAM was $\mathrm{TbFe}_{2}$ 18. Some of its structural features were used for testing the randomly packed hard spheres model corresponding to rare-earth-transition metal alloys. The $\mathrm{Tb}-\mathrm{Tb}$ correlation function, obtained on the basis of generated clusters with spheres of two sizes in Ref. 62 is in qualitative agreement with neutron scattering data on amorphous $\mathrm{TbFe}_{2}$. Using the simple charge model, both classical 62 and quantum mechanical calculations 73 carried out on these clusters show the evidence of the anisotropy term in (13) as well as that the distribution of directions of the local easy axes is effectively isotropic, and indicate the apparent absence of correlation between the directions of axes at neighboring sites.

Magnetic properties of the surroundings of rare-earth atoms are neglected within this model and thus the RAM does not describe correctly alloys of rare-earth - Fe type. Realistic models would have to include both magnetic iron-iron and rareearth - iron interactions. Therefore the RAM is most adequate to describe the intermetallic alloys of rare-earth with non-magnetic compounds.

A considerable number of various experimental data on alloys described by the RAM are listed in an exhaustive review by Cochrane et al. 71. As follows from their analysis, some of these materials order magnetically at sufficiently low temperature, 
but the nature of this ordering is not clear. Among possible low-temperature phases they discuss ferromagnetic ordering, spin-glass phase, quasi long-range ordering. It is clear, that the type of ordering is connected with the alloy intrinsic structure, that corresponds within the model to the distribution of anisotropy axes.

Another interesting application of the RAM is the description of the isotropicnematic phase transition in liquid crystals in porous media. Monte Carlo simulations give the quasi long-range order in this case 74 , while experimental results suggest that the bulk nematic phase is replaced by a "glassy" state 75 .

\section{Effective Hamiltonians and the renormalization group}

In the theory of critical phenomena it is standard to rely on the methods of renormalization group (RG). The basic conjecture of this approach states that a system undergoing a second-order phase transition is scale-invariant at the critical point $T_{c}$. Near to the critical point the behavior of thermodynamic and correlation functions are determined only by the correlation length $\xi$ (c.f. Table I) which describes the scale of the correlations of the order parameter fluctuations.

Among the various mathematical schemes of renormalization group the most accurate numerical results for critical phenomena are obtained in terms of the fieldtheoretical formulation $\mathrm{B}$. In this formulation the universal critical properties of a statistical system are obtained from the large-scale behavior of the corresponding effective field-theoretical Hamiltonian. The latter is treated with the well-elaborated methods of quantum field theory. The relevant global properties of a microscopic model is represented by the structure of the effective Hamiltonian. In particular, the effective Hamiltonian that describes the critical behavior of the Stanley model (1) reads

$$
\mathcal{H}(\vec{\phi})=\int \mathrm{d}^{d} x\left\{\frac{1}{2}\left[|\nabla \vec{\phi}|^{2}+\mu_{0}^{2}|\vec{\phi}|^{2}\right]+\frac{\tilde{u}_{0}}{4 !}|\vec{\phi}|^{4}\right\} .
$$

and is usually referred to as the Hamiltonian of the Landau-Ginzburg-Wilson theory. Here, $\mu_{0}^{2}$ is a bare mass proportional to the temperature difference $\tau$ to the critical point, $\vec{\phi}=\vec{\phi}(\mathbf{R})$ is a $m$-component vector field, $\tilde{u}_{0}$ is a bare coupling. Note, that the effective Hamiltonian (17) preserves the $O(m)$ symmetry of the Stanley model (11).

\subsection{Weakly diluted Stanley model with point-like uncorrelated defects}

A weak quenched disorder term can be introduced directly into the effective Hamiltonian (17). As it was mentioned in Section 2 the presence of non-magnetic impurities in a microscopic model (3) manifests itself in fluctuations of the local temperature of the phase transition. Introducing $\psi=\psi(\mathbf{R})$ as the field of local critical temperature fluctuations, one obtains the effective disordered Hamiltonian 6 :

$$
\mathcal{H}_{\psi}(\vec{\phi})=\int \mathrm{d}^{d} x\left\{\frac{1}{2}\left[|\nabla \vec{\phi}|^{2}+\left(\mu_{0}^{2}+\psi\right)|\vec{\phi}|^{2}\right]+\frac{\tilde{u}_{0}}{4 !}|\vec{\phi}|^{4}\right\} .
$$


The Hamiltonian (18) depends on a number of macroscopic parameters that describe the specific configuration of the field $\psi(\mathbf{R})$. On the other hand, the observables should not depend on the specific realization of the random field $\psi$ and are to be averaged over the possible configurations of $\psi$. . In particular, the singular contribution to the free energy of the diluted quenched Stanley model (3) can be written in the form of a functional integral:

$$
F \propto \int D[\psi(x)] P[\psi(x)] \ln Z[\psi(x)],
$$

where the configurationally-dependent partition function $Z[\psi(x)]$ is the normalizing factor of the Gibbs distribution with effective Hamiltonian (18); $P[\psi]$ defines a probability distribution of the field $\psi(x)$.

In order to avoid the averaging of the logarithm in (19) the standard step is to use the replica trick 7 . This amounts to writing the logarithm in the form of the following limit:

$$
\ln Z=\lim _{n \rightarrow 0} \frac{Z^{n}-1}{n} .
$$

While the powers of $Z$ can only be evaluated for integer values of $n$, analytical continuation in $n$ is assumed to perform the limit $n \rightarrow 0$. Introducing $n$ replicas of the model (18) and taking that $\psi$ obeys a Gaussian distribution:

$$
P(\psi)=\frac{1}{\sqrt{4 \pi} w} \exp \left(-\psi^{2} / 4 \omega^{2}\right)
$$

with $\omega^{2}$ being the dispersion parameter, one ends up 76 with the effective Hamiltonian:

$$
\mathcal{H}(\vec{\phi})=\int \mathrm{d}^{d} x\left\{\frac{1}{2} \sum_{\alpha=1}^{n}\left[\left|\nabla \vec{\phi}^{\alpha}\right|^{2}+\mu_{0}^{2}\left|\vec{\phi}^{\alpha}\right|^{2}\right]+\frac{u_{0}}{4 !} \sum_{\alpha=1}^{n}\left(\left|\vec{\phi}^{\alpha}\right|^{2}\right)^{2}+\frac{v_{0}}{4 !}\left(\sum_{\alpha=1}^{n}\left|\vec{\phi}^{\alpha}\right|^{2}\right)^{2}\right\} .
$$

In the limit $n \rightarrow 0$ the field theory (22) describes the critical properties of the weakly diluted Stanley model. Here, the bare coupling $u_{0}$ is positive (being proportional to $\tilde{u}_{0}$ ) whereas the bare coupling $v_{0}$ is proportional to minus the variance of the random variable $\psi$ and thus it is negative. The last term in (22) is present only for non-zero dilution: it is directly responsible for the effective interaction between replicas due to the presence of impurities 78 .

The model (22) is interesting also in the polymer limit $m \rightarrow 0$. In this case it can be interpreted as a model for SAWs in disordered media. Note, that this limit is not trivial. As noticed by Kim 62, once the limit $m, n \rightarrow 0$ has been taken, both $u_{0}$ and $v_{0}$ terms are of the same symmetry, and an effective Hamiltonian with one coupling $U_{0} \equiv u_{0}+v_{0}$ of $O(m n=0)$ symmetry results. This leads to the conclusion that weak quenched uncorrelated disorder does not change the universal critical properties of SAWs. 


\subsection{Weakly diluted Stanley model with long-range correlated defects}

In the above subsection we obtained an effective Hamiltonian for systems with uncorrelated point-like disorder. Now let us consider the case, when the impurities are long-range-correlated 14 . In particular, let us consider the case, when the inhomogeneities in the system cause fluctuations in the local transition temperature $T_{\mathrm{c}}(\vec{x})$, characterized by the correlation function $g(|\vec{x}-\vec{y}|)=\left\langle T_{\mathrm{c}}(\vec{x}) T_{\mathrm{c}}(\vec{y})\right\rangle-\left\langle T_{\mathrm{c}}(\vec{x})\right\rangle^{2}$, that falls off with distance according to the power law (12). The Fourier-transform $\tilde{g}(k)$ of $g(x)$ reads for small $k$ :

$$
\tilde{g}(k) \sim v_{0}+w_{0} k^{a-d} .
$$

Note, that in the case of random uncorrelated point-like defects the site-occupation correlation function reads: $g(|\vec{x}-\vec{y}|) \sim \delta(\vec{x}-\vec{y})$, so its Fourier transform obeys:

$$
\tilde{g}(k) \sim v_{0} .
$$

As above, we apply the replica trick to average the free energy over different configurations of quenched disorder and construct the effective Hamiltonian of the Stanley model with long-range-correlated disorder 14 :

$$
\begin{gathered}
\mathcal{H}(\vec{\phi})=\int \mathrm{d}^{d} x\left\{\frac{1}{2} \sum_{\alpha=1}^{n}\left[\left|\nabla \vec{\phi}^{\alpha}\right|^{2}+\mu_{0}^{2}\left|\vec{\phi}^{\alpha}\right|^{2}\right]+\frac{u_{0}}{4 !} \sum_{\alpha=1}^{n}\left(\left|\vec{\phi}^{\alpha}\right|^{2}\right)^{2}\right\}+ \\
\sum_{\alpha, \beta=1}^{n} \int \mathrm{d}^{d} x \mathrm{~d}^{d} y g(|\vec{x}-\vec{y}|)\left|\vec{\phi}^{\alpha}(x)\right|^{2}\left|\vec{\phi}^{\beta}(y)\right|^{2} .
\end{gathered}
$$

Here, the notations are as in formula (17), and the replica interaction vertex $g(x)$ is the correlation function with Fourier image (23).

Passing to the Fourier image in (25) and taking into account eq.(23), an effective Hamiltonian results that contains three bare couplings $u_{0}, v_{0}, w_{0}$. Note, that as far as we are interested in the long-wave limit $k \rightarrow 0$, for $a>d$ the $w_{0}$-term in (23) becomes irrelevant and one obtains an effective Hamiltonian of a quenched diluted (short-range correlated) Stanley model 66 with two couplings $u_{0}, v_{0}$. For $a<d$ we have, in addition to the momentum-independent couplings, the momentum dependent one $w_{0} k^{a-d}$. Note, that magnetic systems with parallel extended impurities are not mentioned in our following description; we refer the interested reader to the Ref. 123 for review and numerical results.

The ana|ysis of SAWs in media with long-range-correlated disorder uses Kim's observation 62 that in the limit $m, n \rightarrow 0$ one may pass to an effective Hamiltonian with only two couplings $U_{0}=u_{0}+v_{0}$ and $w_{0}$ (in what follows below we will keep the notation $u_{0}$ for this new coupling $U_{0}$ ). In discrete momentum space this effective Hamiltonian reads 69.70:

$$
\mathcal{H}(\vec{\phi})=\sum_{k} \sum_{\alpha}^{n} \frac{1}{2}\left(\mu_{0}^{2}+k^{2}\right)\left(\vec{\phi}_{k}^{\alpha}\right)^{2}+\frac{u_{0}}{4 !} \sum_{\alpha}^{n} \sum_{k_{1} k_{2} k_{3} k_{4}} \delta\left(\sum_{i=1}^{4} k_{i}\right)\left(\vec{\phi}_{k_{1}}^{\alpha} \vec{\phi}_{k_{2}}^{\alpha}\right)\left(\vec{\phi}_{k_{3}}^{\alpha} \vec{\phi}_{k_{4}}^{\alpha}\right)+
$$




$$
\frac{w_{0}}{4 !} \sum_{\alpha \beta}^{n} \sum_{k k_{1} k_{2} k_{3} k_{4}}|k|^{a-d} \delta\left(\sum_{i=1}^{2} k_{i}+k\right) \delta\left(\sum_{i=3}^{4} k_{i}-k\right)\left(\vec{\phi}_{k_{1}}^{\alpha} \vec{\phi}_{k_{2}}^{\alpha}\right)\left(\vec{\phi}_{k_{3}}^{\beta} \vec{\phi}_{k_{4}}^{\beta}\right), m, n \rightarrow 0 .
$$

Here, the $\delta(k)$ represent products of Kronecker symbols and the notation $(\vec{\phi} \vec{\phi})$ implies a scalar product. Note that the $w_{0}$-term contains the interactions between replicas and an additional power of an internal momentum. Again, it may be shown that for $a=d$ in the limit $m, n \rightarrow 0$ both $u_{0}$ and $w_{0}$ terms are of the same symmetry and one is left with an $O(m n=0)$-vector model with only one coupling $\left(u_{0}+w_{0}\right)$ 70.

\subsection{Stanley model with random anisotropy}

In the cases considered so far, point-like defects introduced random scalar variables to the effective Hamiltonian (17). Random anisotropy disorder on the other hand (17) is different in this respect. The random variable of the disordered effective Hamiltonian for the RAM has the form of a vector with random orientation and given magnitude. Assuming this magnitude as constant one obtains the replicated Hamiltonian as follows

$$
\mathcal{H}_{\hat{\mathbf{z}}}(\vec{\phi})=\int \mathrm{d}^{d} x\left\{\frac{1}{2} \sum_{\alpha=1}^{n}\left[\left|\nabla \vec{\phi}^{\alpha}\right|^{2}+\mu_{0}^{2}\left|\vec{\phi}^{\alpha}\right|^{2}\right]+\frac{u_{0}}{4 !} \sum_{\alpha=1}^{n}\left|\vec{\phi}^{\alpha}\right|^{4}-\sum_{\alpha=1}^{n} D\left(\hat{z} \vec{\phi}^{\alpha}\right)^{2}\right\} .
$$

Here, the notations are as in (17), $D$ is the magnitude of the anisotropy field, $\hat{z}$ is a random unit vector pointing in the direction of the local anisotropy field.

As it was noted above, to perform the average over the random variables $\hat{z}$ we will use the distributions (14) and (15) given in Section 2. For the isotropic distribution of the directions of random anisotropy averaging the axis of all sites (14) results in an effective Hamiltonian of the following form 19

$$
\begin{aligned}
\mathcal{H}(\vec{\phi})= & \int \mathrm{d}^{d} x\left\{\frac{1}{2} \sum_{\alpha=1}^{n}\left[\left|\nabla \vec{\phi}^{\alpha}\right|^{2}+\mu_{0}^{2}\left|\vec{\phi}^{\alpha}\right|^{2}\right]+\frac{u_{0}}{4 !} \sum_{\alpha=1}^{n}\left|\vec{\phi}^{\alpha}\right|^{4}+\right. \\
& \left.\frac{v_{0}}{4 !}\left(\sum_{\alpha=1}^{n}\left|\vec{\phi}^{\alpha}\right|^{2}\right)^{2}+\frac{w_{0}}{4 !} \sum_{\alpha, \beta=1}^{n} \sum_{i, j=1}^{m} \phi_{i}^{\alpha} \phi_{j}^{\alpha} \phi_{i}^{\beta} \phi_{j}^{\beta}\right\},
\end{aligned}
$$

with the bare couplings $u_{0}>0, v_{0}>0, w_{0}<0$. Furthermore, the values of $v_{0}$ and $w_{0}$ are related to $D$ and appropriate cumulants of the distribution function (14) in such a way that their ratio equals $w_{0} / v_{0}=-m$. Note that the symmetry of the $u_{0}$ and $v_{0}$ terms corresponds to the random site Stanley model (22). However the $v_{0}$-term has the opposite sign.

Now, we consider the case of the distribution of $\hat{z}$ along cubic axes (15). After performing the averaging over configurations the corresponding effective Hamiltonian reads 19 :

$$
\mathcal{H}(\vec{\phi})=\int \mathrm{d}^{d} x\left\{\frac{1}{2} \sum_{\alpha=1}^{n}\left[\left|\nabla \vec{\phi}^{\alpha}\right|^{2}+\mu_{0}^{2}\left|\vec{\phi}^{\alpha}\right|^{2}\right]+\frac{u_{0}}{4 !} \sum_{\alpha=1}^{n}\left|\vec{\phi}^{\alpha}\right|^{4}+\right.
$$




$$
\left.\frac{v_{0}}{4 !}\left(\sum_{\alpha=1}^{n}\left|\vec{\phi}^{\alpha}\right|^{2}\right)^{2}+\frac{w_{0}}{4 !} \sum_{i=1}^{m} \sum_{\alpha, \beta=1}^{n} \phi_{i}^{\alpha 2} \phi_{i}^{\beta^{2}}+\frac{y_{0}}{4 !} \sum_{i=1}^{m} \sum_{\alpha=1}^{n} \phi_{i}^{\alpha 4}\right\},
$$

where the bare couplings are $u_{0}>0, v_{0}>0, w_{0}<0$. The symmetry of the $w_{0}$ terms in (29) differs from that in (28). Furthermore, the values of $w_{0}$ and $v_{0}$ differ for the Hamiltonians (28) and (29) due to the cubic distribution (15) but their ratio equals $-m$ again. The last term in (29) combines the symmetries of terms with coefficients $u_{0}$ and $w_{0}$. It does not result from the functional representation of the free energy but is generated by further application of the RG transformation. Therefore $y_{0}$ can be of either sign.

\subsection{Field-theoretical renormalization-group approach}

In this subsection, we give a brief account of the main relations of the fieldtheoretical renormalization group ( $\mathrm{RG}$ ) formalism that can be evaluated in different variants. We choose the massive field theory scheme with renormalization of the one-particle irreducible vertex functions $\Gamma_{0}^{(L, N)}\left(k_{1}, . ., k_{L} ; p_{1}, . ., p_{N} ; \mu_{0}^{2} ;\left\{\lambda_{0}\right\}\right)$ at non-zero mass and zero external momenta 79 . The one-particle irreducible vertex function can be defined as:

$$
\begin{aligned}
& \delta\left(\sum k_{i}+\sum p_{j}\right) \Gamma_{0}^{(L, N)}\left(\{k\} ;\{p\} ; \mu_{0}^{2} ;\left\{\lambda_{0}\right\}\right)=\int^{\Lambda_{0}} e^{i\left(k_{i} R_{i}+p_{j} r_{j}\right)} \times \\
& \left\langle\phi^{2}\left(r_{1}\right) \ldots \phi^{2}\left(r_{L}\right) \phi\left(R_{1}\right) \ldots \phi\left(R_{N}\right)\right\rangle_{1 P I}^{\mathcal{H}_{\text {eff }}} \mathrm{d}^{d} R_{1} \ldots \mathrm{d}^{d} R_{N} \mathrm{~d}^{d} r_{1} \ldots \mathrm{d}^{d} r_{L} .
\end{aligned}
$$

Here, $\left\{\lambda_{0}\right\}$ stands for the set of bare couplings of the effective Hamiltonian, $\{p\},\{k\}$ are the sets of external momenta, $\Lambda_{0}$ is the cutoff, and the averaging is performed with the corresponding effective Hamiltonian, $\mathcal{H}_{\text {eff }}$.

An intrinsic feature of the evaluation scheme (30) are divergences in the limit $\Lambda_{0} \rightarrow \infty$. In order to remove them and to map the divergent mathematical objects to convergent physical quantities one performs a controlled rearrangement of the series for the vertex functions. Introducing renormalizing factors for the fields $Z_{\phi}$, $Z_{\phi^{2}}$, the renormalized vertex functions $\Gamma_{R}^{(L, N)}$ are expressed in terms of the bare vertex functions as follows:

$$
\Gamma_{R}^{(L, N)}\left(\{k\} ;\{p\} ; \mu^{2} ;\{\lambda\}\right)=Z_{\phi^{2}}^{L} Z_{\phi}^{N / 2} \Gamma_{0}^{(L, N)}\left(\{k\} ;\{p\} ; \mu_{0}^{2} ;\left\{\lambda_{0}\right\}\right),
$$

where $\mu,\{\lambda\}$ are the renormalized mass and couplings.

The regularization scheme suggested in Eq. (31) is not unique. To define the regularization scheme completely one imposes renormalization conditions for the renormalized vertex functions. In the massive scheme the renormalization conditions read 29 :

$$
\begin{aligned}
\left.\Gamma_{R}^{(0,2)}\left(k,-k ; \mu^{2},\{\lambda\}\right)\right|_{k=0} & =\mu^{2}, \\
\left.\frac{d}{d k^{2}} \Gamma_{R}^{(0,2)}\left(k,-k ; \mu^{2},\{\lambda\}\right)\right|_{k=0} & =1,
\end{aligned}
$$




$$
\begin{aligned}
\left.\Gamma_{R, \lambda_{j}}^{(0,4)}\left(\{k\} ; \mu^{2},\{\lambda\}\right)\right|_{k=0} & =\mu^{4-d} \lambda_{j}, \\
\left.\Gamma_{R}^{(1,2)}\left(p ; k,-k ; \mu^{2},\{\lambda\}\right)\right|_{k=p=0} & =1 .
\end{aligned}
$$

In the case of long-range-correlated disorder we have another global parameter $a$ along with $d$, and for the renormalization of the coupling $w$ one imposes 80 :

$$
\left.\Gamma_{R, w}^{(0,4)}\left(\{k\} ; \mu^{2},\{\lambda\}\right)\right|_{k=0}=\mu^{4-a} w
$$

with the renormalized coupling $w$. The scaling properties of the system asymptotically close to the critical point are expressed by the homogeneous Callan-Symanzik equations 3 for $\Gamma_{R}^{(L, N)}\left(\{p\} ;\{k\} ; \mu^{2},\{\lambda\}\right)$ :

$$
\begin{aligned}
\left\{\mu \frac{\partial}{\partial \mu}+\right. & \sum_{i} \beta_{\lambda_{i}}(\{\lambda\}) \frac{\partial}{\partial \lambda_{i}}- \\
& \left.\left(\frac{N}{2}-L\right) \gamma_{\phi}(\{\lambda\})+L \bar{\gamma}_{\phi^{2}}(\{\lambda\})\right\} \Gamma_{R}^{(L, N)}\left(\{p\} ;\{k\} ; \mu^{2},\{\lambda\}\right)=0,
\end{aligned}
$$

with the coefficients:

$$
\beta_{\lambda_{i}}(\{\lambda\})=\left.\frac{\partial \lambda_{i}}{\partial \ln \mu}\right|_{\left\{\lambda_{0}\right\}, \mu_{0}}, \gamma_{\phi}=\left.\frac{\partial Z_{\phi}}{\partial \ln \mu}\right|_{\left\{\lambda_{0}\right\}, \mu_{0}}, \bar{\gamma}_{\phi^{2}}=-\left.\frac{\partial \bar{Z}_{\phi^{2}}}{\partial \ln \mu}\right|_{\left\{\lambda_{0}\right\}, \mu_{0}}
$$

where $\bar{Z}_{\phi^{2}}=Z_{\phi^{2}} Z_{\phi}$.

The fixed points $\left\{\lambda^{*}\right\}$ of the RG transformation are given by the solution of the system of equation:

$$
\beta_{\lambda_{i}}\left(\left\{\lambda^{*}\right\}\right)=0, \quad i=1,2, \ldots
$$

The stable fixed point, corresponding to the critical point of the system, is defined as the fixed point where the stability matrix:

$$
B_{i j}=\frac{\partial \beta_{\lambda_{i}}}{\partial \lambda_{j}}
$$

possesses eigenvalues $\left\{\omega_{i}\right\}$ with positive real parts. In this point, the function $\gamma_{\phi}$ determines the value of the pair correlation function critical exponent $\eta$ :

$$
\eta=\gamma_{\phi}\left(\left\{\lambda^{*}\right\}\right),
$$

and the correlation length critical exponent $\nu$ is determined as:

$$
\nu^{-1}=2-\gamma_{\phi}\left(\left\{\lambda^{*}\right\}\right)-\bar{\gamma}_{\phi^{2}}\left(\left\{\lambda^{*}\right\}\right) .
$$

All other critical exponents may be obtained from familiar scaling laws. For example, the susceptibility exponent $\gamma$ is given by:

$$
\gamma=\nu(2-\eta) .
$$

The $\beta$ - and $\gamma$-functions are calculated perturbatively as series in the (several) couplings $\lambda_{i}$. The order of the expansion corresponds to the number of loops in the diagrammatic Feynman representation of the vertex functions (30). However, due to the (asymptotic) divergence of the RG functions series, one cannot directly derive the reliable physical information from these expressions. The methods that permit to cope with the task will be described in the next section. 


\section{Resummation of asymptotic series}

The expansions of RG functions in powers of couplings (weak coupling expansions) are known for a number of models with high accuracy. However, the very idea of perturbation theory is that the result is successively approximated by accounting higher order contributions. But the method seems doomed to failure if the series are divergent. This is the case in the field-theoretical renormalization group approach. Moreover, here the series are characterized by a factorial growth of the coefficients implying a zero radius of convergence. A simple method to manage this problem is optimally truncating the series, i.e. accounting only its first several terms that do not show the divergence. A self-consistent way to take into account the higher order contributions requires the application of special ways of resummation: Borel

resummation accompanied by certain additional procedures 81. Resumming a series one should note that the accuracy of the resummed results depends on the procedure of resummation. Thus the analysis and appropriate selection of an adapted resummation procedure is crucial to obtain reliable results.

However, there remains the principal question about the Borel summability of the perturbation theory series for a given model. Up to now this has been proved only for the $\phi^{4}$ theory with one coupling 82 . For years, the field theoretical RG series for models with several couplings were analyzed as if they are asymptotically divergent without a proof of this property. Moreover, there exists strong evidence of possible Borel non-summability of the series obtained for disordered models 83.84 .85 . In this section, we will give the definition of an asymptotic series and provide the methods which currently are used to extract convergent data.

\subsection{An asymptotic series}

For a function $f(g)$ given in the form of a power series

$$
f(g)=\sum_{k=0}^{\infty} a_{k} g^{k}
$$

the basic definition of its asymptotic nature is:

$$
\left|f(g)-\sum_{k=0}^{N} a_{k} g^{k}\right| \leq a_{k+1} g^{k+1}
$$

for fixed $N$ and small $g$.

In general, the nature of an asymptotic series is such that the correct sum is uniformly approached until, after an optimum number $N_{0}$ of terms the subsequent terms drive the partial sums away from the correct result for the total sum, leading to divergence 81 .

For models with a simple $O(m)$-symmetry the factorial growth of the RG functions coefficients for large orders of perturbation theory is well established 86 :

$$
a_{k}=k !(-a)^{k} k^{b} c\left[1+O\left(\frac{1}{k}\right)\right], \quad k \rightarrow \infty
$$


the quantities $a$ and $b$ have been calculated in Ref. 87 , the values of constant $c$ in Ref. 8 . The estimate (41) is a fundamental one since it shows that the initial functions can be reconstituted from their asymptotic expansions by the application of the integral Borel transformation 22 in different modifications. As it was already noted above the asymptotic nature of the divergence of the RG functions for the disordered models still has not been proved.

Below we will describe the methods of resummation which in the field-theoretical RG scheme are applied to study the critical behavior of the Stanley model (17) as well as the disordered models (22), (25), (26), (28), (29).

\subsection{Resummation of asymptotic series with one coupling}

The idea of resummation which can be applied to an asymptotic series consists in changing the order of summation 81 . In the case of one variable it means that starting from a power series

$$
S(x)=\lim _{L \rightarrow \infty} \sum_{i=1}^{L} a_{i} x^{i},
$$

one performs an identical transformation 39

$$
\begin{aligned}
& S(x) \equiv \lim _{L \rightarrow \infty} \sum_{i=1}^{L}\left(\frac{a_{i} x^{i}}{i !} \int_{0}^{\infty} d t \exp (-t) t^{i}\right) \equiv \\
& \lim _{L \rightarrow \infty}\left\{\lim _{A \rightarrow \infty} \int_{0}^{A} d t\left[\sum_{i=1}^{L} \frac{a_{i}(x t)^{i}}{i !} \exp (-t)\right]\right\},
\end{aligned}
$$

and redefines the sum by the following expression:

$$
\begin{gathered}
S^{\prime}(x)=\lim _{A \rightarrow \infty}\left\{\lim _{L \rightarrow \infty} \int_{0}^{A} d t\left[\sum_{i=1}^{L} \frac{a_{i}(x t)^{i}}{i !} \exp (-t)\right]\right\} \equiv \\
\int_{0}^{\infty} d t \exp (-t) \sum_{i=1}^{\infty} \frac{a_{i}(x t)^{i}}{i !},
\end{gathered}
$$

where $\sum_{i=1}^{L} a_{i}(x t)^{i} / i$ ! is called the Borel - image of $\sum_{i=1}^{L} a_{i} x^{i}$. This trick is natural in the sense that for the case of a convergent series one finds $S^{\prime}=S$ within the radius of convergence.

The above mentioned procedure in the case of a single variable is known as the Borel resummation technique and in different modifications it is widely used for evaluating asymptotic series. Unfortunately this technique cannot be applied to our case immediately because only the partial sum of the truncated series is known. To overcome this obstacle one represents the Borel-image of the initial sum in the form of a rational approximant and in this way reconstitutes the general form of the series. This technique that involves a rational approximation and the Borel transformation is known as the Padé-Borel resummation technique 90 and is performed as follows:

- the Borel-image of the initial sum is constructed:

$$
\sum_{i=1}^{L} a_{i} x^{i} \Rightarrow \sum_{i=1}^{L} \frac{a_{i}(x t)^{i}}{i !}
$$


- the Borel-image is extrapolated by a rational approximant

$$
[M / N]=[M / N](x t) ;
$$

here, $[M / N]$ stands for the quotient of two polynomials in $x t ; M$ is the order of the numerator and $N$ is that of the denominator;

- the resummed function is then obtained by the integral:

$$
S^{r e s}(x)=\int_{0}^{\infty} d t \exp (-t)[M / N](x t) .
$$

The Padé-Borel procedure can be generalized by introducing an additional fit parameter $p$ to the Borel transformation. Substituting the factorial $i$ ! by the Euler gamma-function $\Gamma(i+p+1)$ and inserting an additional factor $t^{p}$ into the integral (47), one defines the Padé-Borel-Leroy resummation procedure.

The Padé-Borel as well as Padé-Borel-Leroy resummation procedures are applicable to RG functions of one coupling constant such as those of model (17). For models with more complicated symmetries and several couplings one uses the generalizations of the Padé-Borel method as explained in the following subsection.

\subsection{The case of several couplings}

In order to suit the resummation procedure (45)-(47) for functions that depend on several variables one should change the first step (45): for example, for the two-variable case one defines the Borel image by 91 :

$$
\sum_{i, j} a_{i, j} x^{i} y^{j} \Rightarrow \sum_{i, j} \frac{a_{i, j}(x t)^{i}(y t)^{j}}{(i+j) !} .
$$

The rational approximation (46) can be performed then either in the dummy variable $t$ or in the variables $x, y$. This defines two approaches that one can use to resum the functions of several variables. In the first case the resummation procedure is referred to as the Padé-Borelresummation for resolvent series 92 . The application of the Chisholm approximants 93 which are the generalization of Padé-approximants to the many-variable case is necessary in the second case. A Chisholm approximant can be defined as a ratio of two polynomials both in variables $x$ and $y$, of degree $M$ and $N$ such that the first terms of its expansion are equal to those of the function which is approximated. Again, the resummation is performed in eq. (47) replacing the Padé with the Chisholm approximant. This method will be referred below as the Chisholm-Borel resummation.

It is obvious that an initial sum can be resummed in different ways. Apart from the Leroy fit parameter $p$ mentioned above some arbitrariness arises from the different types of rational approximants one may construct. For instance, within the two-loop approximation the method of Padé-Borel resummation of a resolvent series can be done using either the $[0 / 2]$ or the $[1 / 1]$ approximants. The ChisholmBorel approximation implies even more arbitrariness and demands a very precise analysis of the approximants to be chosen. 
We want to stress here again that in contrast to the single variable case the validity of neither the Padé-Borel nor the Chisholm-Borel resummations have been proven rigorously for any cases with two or more variables. The success of their application can solely be judged by the consistency of the results with both experimental and Monte-Carlo data.

\subsection{The method of subsequent resummation}

In the resummation schemes described above for series in several variables the variables are treated as "equal in rights". This is not the case in the recently proposed method of so-called subsequent resummation, developed in the context of the $d=0$-dimensional disordered Ising model in Ref. 85 .

Starting from the RG function $f(u, v)$ in the form of a series in powers of $u, v$ (for the model (22), $u$ is the original coupling of the undiluted system and $v$ is the variance of the quenched disorder)

$$
f(u, v)=\sum_{n} \sum_{k} C_{k, n} u^{k} v^{n}
$$

one rewrites it as a series in the variable $v$ :

$$
f(u, v)=\sum_{n} A_{n}(u) v^{n}
$$

with coefficients that are in turn series in the variable $u$ and are to be resummed in advance by any one-variable method:

$$
A_{n}(u) \equiv \sum_{k} C_{k, n} u^{k}
$$

The main result of Ref. 85 is that the expansions of the coefficients (51) and the resulting series (50) at fixed $u$ are Borel summable (for a $d=0$ dimensional system, however). This gives a hint to analyze the RG expansions of the $d=3$ disordered models by first performing a Padé-Borel resummations of the corresponding series for the coefficients in one coupling and then, using the computed coefficients, resumming the series in the other subsequent coupling(s).

\section{Results of the renormalization group analysis}

Now, having at hand the powerful methods of the field-theoretical renormalization group refined by resummation of the (asymptotic) series we can check to what results does it lead when applied to systems with weak quenched disorder. Again, we start from the uncorrelated point-like disorder, then pass to the systems with long-range correlated disorder, and finally we analyze random-anisotropy systems. 


\subsection{Weakly diluted Stanley model with point-like uncorrelated defects}

Referring to the universal critical properties of the model 22 at $d=3$, as implied by the Harris criterion one observes new critical behavior only for the case $m=1$, i.e. for the weakly diluted quenched Ising model.

Following the procedure sketched in the subsection 4.4 the expressions for the RG functions of the model are obtained as series in the renormalized couplings $u$ and $v$. Each order of the perturbation theory corresponds to a given number of integrations in momentum space and, equivalently, to the number of loops in Feynman diagrams in the diagrammatic representation for the vertex functions (30).

The massive RG functions of the weakly diluted quenched Ising model are known to high orders of the perturbation theory. They result from more than twenty years of laborious studies. Within the three-loop accuracy the functions were first reported in Ref. 94. They yet contained errors which were corrected in Ref. 95, erroneously again. The final three-loop expressions were only published in Ref. 96 . Subsequently, four-loop series were obtained in Ref. 97. Recently five-loop 98 and record six-loop 99 expansions became available. Written in the two-loop approximation the functions read 100 :

$$
\begin{aligned}
\beta_{u}(u, v)= & -(4-d) u\left\{1-u-\frac{3}{2} v+\frac{8}{27}\left[9\left(i_{1}-\frac{1}{2}\right)+i_{2}\right] u^{2}+\right. \\
& \left.\frac{2}{3}\left[12\left(i_{1}-\frac{1}{2}\right)+i_{2}\right] u v+\frac{1}{4}\left[21\left(i_{1}-\frac{1}{2}\right)+i_{2}\right] v^{2}\right\}, \\
\beta_{v}(u, v)= & -(4-d) v\left\{1-v-\frac{2}{3} u+\frac{1}{4}\left[11\left(i_{1}-\frac{1}{2}\right)+i_{2}\right] v^{2}+\right. \\
& \left.\frac{2}{3}\left[6\left(i_{1}-\frac{1}{2}\right)+i_{2}\right] v u+\frac{8}{27}\left[3\left(i_{1}-\frac{1}{2}\right)+i_{2}\right] u^{2}\right\}, \\
\gamma_{\phi}(u, v)= & -2(4-d)\left\{\left[\frac{2}{27} u^{2}+\frac{1}{6} u v+\frac{1}{16} v^{2}\right] i_{2}\right\}, \\
\bar{\gamma}_{\phi^{2}}(u, v)= & (4-d)\left\{\frac{1}{3} u+\frac{1}{4} v-12\left[\frac{1}{27} u^{2}+\frac{1}{12} u v+\frac{1}{32} v^{2}\right]\left(i_{1}-\frac{1}{2}\right)\right\} .
\end{aligned}
$$

Here, we use the conventional normalization for the couplings $u, v$ such that the coefficients of $u$ and $v$ in the one-loop contribution to $\beta_{u}$ and $\beta_{v}$ equal unity.

The expressions (52)-(55) besides the couplings $u, v$ depend on space dimension $d$. In particular, $d$ enters the two-loop integrals $i_{1}(d), i_{2}(d)$. The two-loop contributions to the RG functions of the three-dimensional weakly diluted quenched Ising model can be obtained from the expressions (52)-(55) by substituting $i_{1}(3)=2 / 3$, $i_{2}(3)=-2 / 27101$.

The explicit dependence of the functions (52)-(55) on the space dimension $d$ was exploited in Ref. 100. Expanding the values of the loop-integrals $i_{1}, i_{2}$ in $\varepsilon=4-d$, the functions were obtained in the form of an $\varepsilon$-expansion 6 . Classically, this result follows the familiar $\varepsilon$-expansion 102 of the critical exponents. In order to obtain the expansions, one solves the fixed point equations (36) with $\varepsilon$ as a small parameter 
and then re-expands in $\varepsilon$ the critical exponents after substitution of the coordinates $u^{*}(\varepsilon), v^{*}(\varepsilon)$ into the $\gamma$-functions.

This form of analysis of the six-loop massive RG functions encounters hardships since the $\varepsilon$-expansions of the loop integrals are not known in high four-, five- and six-loop approximations. This obstacle can be overcome by applying an alternative dimensional regularization scheme with minimal subtraction 103 . In this approach the RG functions were obtained in the three-loop 104 approximation and can be reconstityted within the five-loop accuracy from the RG functions of the cubic model 105. The degeneracy of the one-loop RG functions lead to the result, that instead of expanding in $\varepsilon$ one is to expand in $\sqrt{\varepsilon} 76,06$. This results in $\sqrt{\varepsilon}$ expansions for the critical exponents 107 and the stability matrix (37) eigenvalues 108 :

$$
\begin{aligned}
\nu & =0.5+0.08411582 \varepsilon^{1 / 2}-0.01663203 \varepsilon+0.04775351 \varepsilon^{3 / 2}+0.27258431 \varepsilon^{2}, \\
\eta & =-0.00943396 \varepsilon+0.03494350 \varepsilon^{3 / 2}-0.04486498 \varepsilon^{2}+0.02157321 \varepsilon^{5 / 2}, \\
\gamma & =1+0.16823164 \varepsilon^{1 / 2}-0.02854708 \varepsilon+0.07882881 \varepsilon^{3 / 2}+0.56450490 \varepsilon^{2}, \\
\omega_{1} & =2 \varepsilon+3.70401119 \varepsilon^{3 / 2}+11.30873837 \varepsilon^{2} \\
\omega_{2} & =0.67292659 \varepsilon^{1 / 2}-1.92550909 \varepsilon-0.57252518 \varepsilon^{3 / 2}-13.93125952 \varepsilon^{2} .
\end{aligned}
$$

In the above expressions the two-loop results for the exponents were obtained in Ref. 66, the three-loop results were presented independently in Refs. 109 and 110.

The crucial property of the $\sqrt{\varepsilon}$-expansions $(56)-(60)$ is their formal character. Though they allow to predict qualitatively new critical behavior in the weakly diluted Ising model 6100 they seem to be of no use for any quantitative analysis. Naively adding the successive perturbational contributions in the $\sqrt{\varepsilon}$-expansion for the model stability matrix eigenvalues (59), (60) one observes that already in the three-loop approximation $(\sim \varepsilon) \omega_{2}$ hecomes negative and therefore no stable fixed point exists in strict $\sqrt{\varepsilon}$-expansion 108 . Even resummation procedures do not change this picture 52.11 .

The way to cope with the bad convergence properties of the $\sqrt{\varepsilon}$-expansion can be found in the fixed-dimension approach which does not imply expanding the loop integrals in $\varepsilon$. This method yields most numerical results for the universal characteristics of the diluted Ising model at criticality. The numerical solution of the fixed point equations (36) the resummed $\beta$-functions (52) and the resummation of the $\gamma$ functions (55) in the stable fixed point shows the validity of the method. The study of the massive $\beta$-functions of the diluted Ising model resummed in this way revealed that starting from the two-loop approximation the random fixed point is stable and

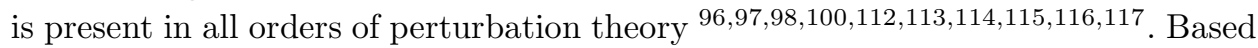
on the three-dimensional values of the loop-integrals 101, the RG functions of the model are calculated 99 and resummed 118 at present in the six-loop approximation.

Let us analyze the fixed point structure of the $\beta$-functions for the random Ising model at $d=3$. Within the one-loop approximation (i.e. including only linear terms in $u, v$ in the curly brackets in (52), (53)) at $d=3$ one finds three fixed points (see Fig. [) : the Gaussian fixed point $\mathbf{G} u^{*}=v^{*}=0$, the pure Ising fixed point $\mathbf{I}$ 


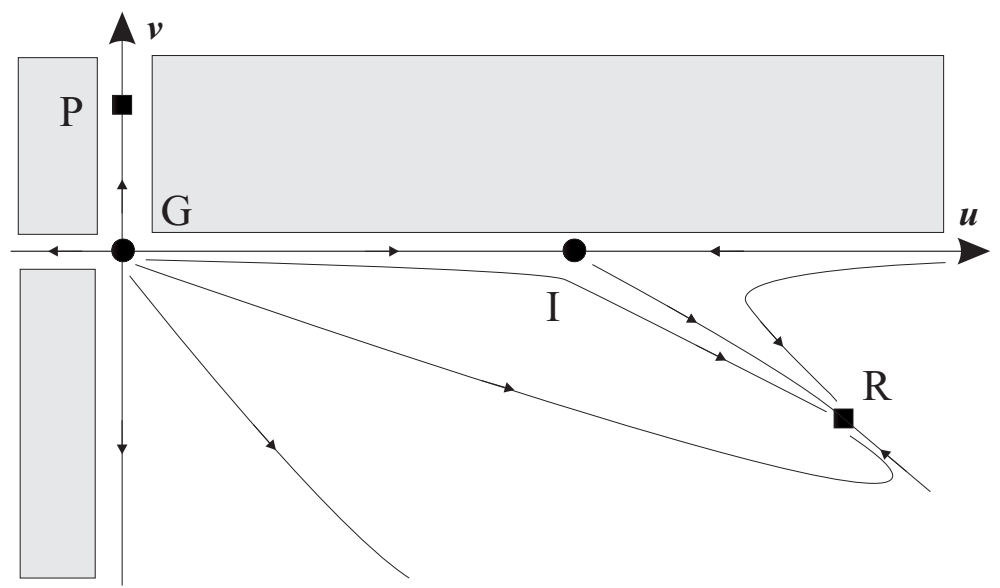

Fig. 6. Qualitative RG flow for the weakly diluted quenched Ising model. The Gaussian fixed point $\mathbf{G}$ is stable for $d \geq 4$, stable fixed point $\mathbf{P}$ can not be reached from the initial coupling values $u>0, v<0$. The unphysical regions for the random Ising model are shown in grey tone. The pure Ising fixed point $\mathbf{I}$ is unstable whereas random fixed point $\mathbf{R}$ is both stable and accessible (stable fixed points are shown by boxes).

$u^{*} \neq 0, v^{*}=0$ and the "polymer" fixed point $\mathbf{P} u^{*}=0, v^{*} \neq 0$. It is straightforward to check that the fixed points $\mathbf{G}$ and $\mathbf{I}$ are unstable whereas the fixed point $\mathbf{P}$ is stable but as far as $v^{*}>0$ it is inaccessible for the initial values of couplings of our model. Within the one-loop approximation one does not encounter the fixed point $\mathbf{R}$ with both non-zero coordinates $u^{*} \neq 0, v^{*} \neq 0$ : this happens because the system of equations for the fixed points is degenerate on the one-loop level 76.106. As we will see, this fixed point appears in the next, two-loop approximation if the resummation is applied.

Applying the Chisholm-Borel resummation procedure of the subsection 5.3 one encounters the random fixed point $\mathbf{R}$ of the model in the two-loop approximation (see Fig. 6). The stability analysis shows that this fixed point is stable proving the crossover to a new critical regime under dilution. In Figs. 7 , 8 we show the curves $\beta_{u}(u, v)=0, \beta_{v}(u, v)=0$ in the $u-v$ plane. The intersections of these curves (i.e. simultaneous zeros of both $\beta$-functions) correspond to the fixed points. The "naive" analysis of the $\beta$-functions, without applying any resummation procedure leads to the curves, which are shown on the left-hand side of the Figs. 77, 8. Without resummation only in the three-loop approximation one finds a stable random fixed point $u^{*} \neq 0, v^{*} \neq 0$. However the fixed point then disappears in the four-loop approximation. A completely different picture is observed when the resummation procedure is applied (right hand columns of the figures). In the region of interest for the values of the couplings the topology of the fixed point picture remains stable for increasing orders of the approximation from the two-loop to the four-loop level. 


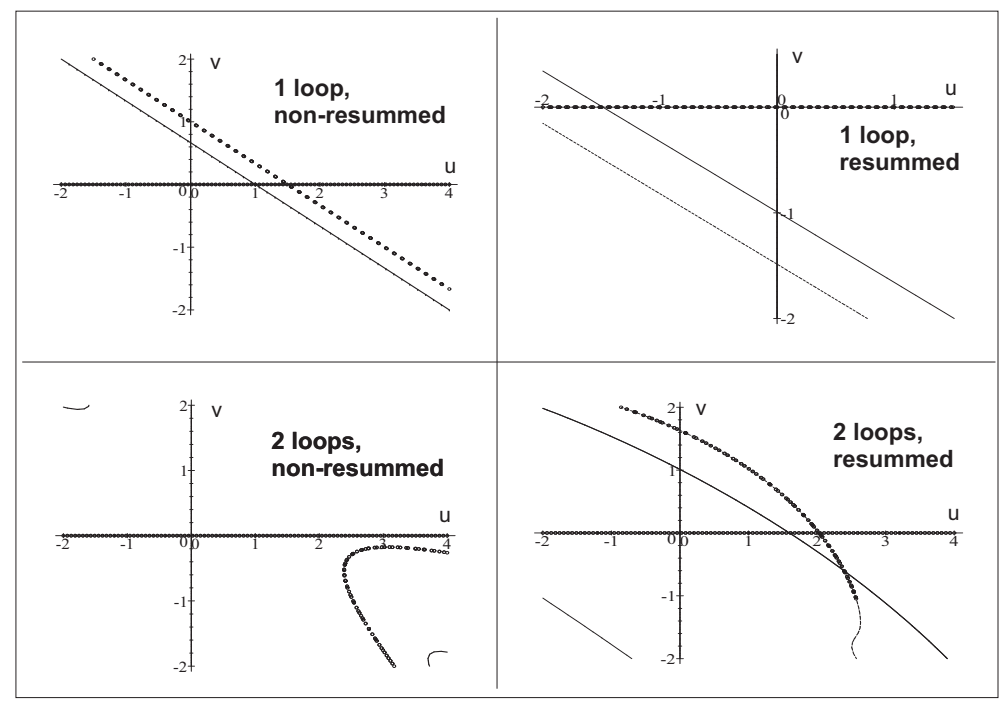

Fig. 7. The lines of zeros of the diluted Ising model massive $\beta$-functions in the one- and two-loop approximations of the perturbation theory, left-hand column: non-resummed, right-hand column: resummed by the Chisholm-Borel method. Circles correspond to $\beta_{u}=0$, thick lines depict $\beta_{v}=0$. Thin solid and dashed lines show the zeros of the analytically continued functions $\beta_{u}$ and $\beta_{v}$ respectively. One can see the appearance of the mixed fixed point $u>0, v<0$ in the two-loop approximation for the resummed $\beta$-functions. The figure is taken from Ref. 116.

The Figs. 7, 8 show also the historical evolution of the study of the weakly diluted quenched Ising model. Already in the two-loop level the RG functions resummed by the Chisholm-Borel procedure 112 revealed that no difficulties connected to the degeneracy of the $\beta$-functions are encountered. Critical exponents extracted from the resummed $\gamma$-functions values in the fixed point were found to be clearly larger than those of the pure model (see Table 3). As noted above, in the three-loop level the straightforward analysis of the $\beta$-functions 9495 yields fixed point coordinates and critical exponents without resummation, but the accuracy obtained did not allow to estimate, for instance the heat capacity critical behavior. Numerical values of the random Ising model critical exponents as obtained by the RG approach are given in Table 3 with reference to the renormalization schemes and resummation procedures 119. We note here a breakthrough that occurred during several months of 1999-2000 when the perturbation theory expansions were extended from the 4th 97 through 5 th 98 to the sixth order 99.118.

Already the 5th loop order Padé-Borel resummation meets some difficulties. Moreover, the extension to the six-loop order revealed a wide gap between the five- and six-loop fixed point coordinates 117 and subsequently an inconsistency of the six-loop values of critical exponents compared with the five-loop results of Refs 98. One also encounters a similar behavior of the resummed series when the $d=3$ minimal subtraction RG technique is applied to the random Ising model: 


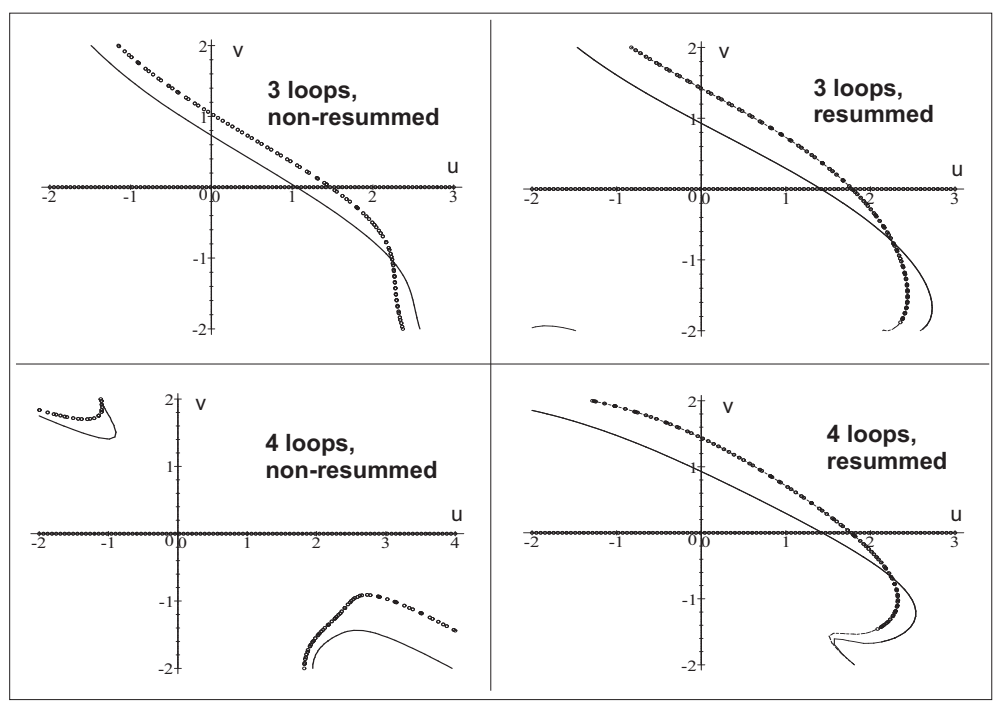

Fig. 8. The lines of zeros of the diluted Ising model massive $\beta$-functions in the three- and fourloop approximations of the perturbation theory, left-hand column: non-resummed, right-hand column: resummed by the Chisholm-Borel method. The notations are the same as in the figure 7. Close to the mixed fixed point the behaviour of the resummed functions remains alike with the increasing order of appximation. This is not the case for the non-resummed functions. The figure is taken from Ref. 116 .

the random fixed point is present in the resummed two- three- andfour- loop approximations whereas it disappears in the five-loop approximation 52 111. This lead to the conjecture about the possible Borel-non-summability of the series.

The fact that the weak coupling expansion might not be of asymptotic nature for the random Ising model was predicted already in a study of the randomly diluted model in zero dimensions 4 . The perturbation theory series of this toymodel appeared to be Borel non-summable. Moreover, this property was shown to be a direct consequence of the appearance of Griffiths-like singularities 33 that are caused by the zeroes of the partition function of the pure system.

However, as noted above, in another recent study of the $d=0$ dimensional random Ising model it was shown analytically 8 that the perturbative expansions for the free energy are Borel summable provided the summation is carried out subsequently, as described above in subsection 5.4. The application of this procedure to the case $d=3$ reconstituted 118 earlier data for the asymptotic critical exponents of the model in massive scheme and allowed to restore the presence of the random fixed point in the $d=3$ minimal subtraction scheme on the five loop level 120 .

Alternatively, the $d=3$ random Ising model was treated non-perturbatively first by means of the Golner-Riedel scaling field approach 121, and very recently by an RG approach based on the concept of effective average action 122 . 
November 9, 2018 10:37 WSPC/Guidelines lecture

32 Yu. Holovatch, V. Blavats'ka, M. Dudka, C. von Ferber, R. Folk, and T. Yavors'kii

Table 3. The theoretical values for the asymptotic critical exponents of the weakly diluted Ising model. $n \mathrm{LA}$ denotes the $n$th order in loopwise approximation within the massive ('mass') and $3 d$ minimal subtraction ('MS') schemes of the field-theoretical renormalization group approach. The resummation procedures are given in the following notations: $\mathrm{ChBr}-$ Chisholm-Borel; $\mathrm{PdBr}-$ Padé-Borel; AW $-\varepsilon$ algorithm of Wynn, CM - Borel transformation with conformal mapping. SF stands for Golner-Riedel scaling field method, EEA denotes nonperturbative RG approach based on the concept of effective average action, superscript ${ }^{c}$ at the correction-to-scaling exponent $\omega$ indicates that the real part of the corresponding complex number is shown.

\begin{tabular}{|c|c|c|c|c|c|c|c|}
\hline Ref. & $\begin{array}{c}\mathrm{RG} \\
\text { scheme }\end{array}$ & Order & $\begin{array}{l}\text { Resum- } \\
\text { mation }\end{array}$ & $\nu$ & $\eta$ & $\gamma$ & $\omega$ \\
\hline $\begin{array}{l}\text { Sokolov et thef } \\
\text { 1981, Ref. } 55\end{array}$ & mass & $3 \mathrm{LA}$ & $\overline{\text { No }}$ & & 0.009 & 1.31 & \\
\hline $\begin{array}{l}\text { Newman et } \\
\text { 1982, Ref. } 121 \text { ' }\end{array}$ & $\overline{\mathrm{SF}}$ & No & & 0.70 & 0.015 & 1.39 & 0.41 \\
\hline $\begin{array}{l}\text { Jug, } 1883, \\
\text { Ref. } 112,\end{array}$ & mass & $2 \mathrm{LA}$ & $\mathrm{ChBr}$ & 0.678 & 0.031 & 1.336 & $0.450^{c}$ \\
\hline $\begin{array}{l}\text { Mayer et } 1+9 . \\
\text { 1984, Ref. } 113\end{array}$ & mass & $\begin{array}{l}2 \mathrm{LA} \\
3 \mathrm{LA}\end{array}$ & $\begin{array}{l}\mathrm{ChBr} \\
\mathrm{ChBr}\end{array}$ & & $\begin{array}{l}0.031 \\
0.022\end{array}$ & $\begin{array}{l}1.337 \\
1.325\end{array}$ & \\
\hline $\begin{array}{l}\text { Mayer et at. } \\
\text { 1989, Ref. }\end{array}$ & mass & $4 \mathrm{LA}$ & $\mathrm{ChBr}$ & 0.670 & 0.034 & 1.326 & \\
\hline $\begin{array}{c}\text { Mayer, } 1989, \\
\text { Ref. } 114,\end{array}$ & mass & $\begin{array}{l}4 \mathrm{LA} \\
4 \mathrm{LA}\end{array}$ & $\begin{array}{c}\mathrm{AW} \\
\mathrm{PdBr}\end{array}$ & $\begin{array}{l}0.6680 \\
0.6714\end{array}$ & & $\begin{array}{l}1.318 \\
1.321\end{array}$ & \\
\hline $\begin{array}{c}\text { Shpot, } 989, \\
\text { Ref. } 96\end{array}$ & mass & $3 \mathrm{LA}$ & $\mathrm{ChBr}$ & 0.671 & 0.021 & 1.328 & 0.359 \\
\hline \begin{tabular}{l|l} 
Janssen et \\
1995, Ref. 104
\end{tabular} & MS, 3d & $3 \mathrm{LA}$ & $\mathrm{PdBr}$ & 0.666 & & 1.313 & 0.366 \\
\hline $\begin{array}{c}\text { Holovatch et.21, } \\
\text { 1997, Ref. } 116]\end{array}$ & mass & $3 \mathrm{LA}$ & $\mathrm{ChBr}$ & 0.671 & 0.019 & 1.328 & 0.376 \\
\hline $\begin{array}{l}\text { Folk et at } \\
\text { 1998, Ref. }\end{array}$ & MS, 3d & $\begin{array}{l}\text { LA } \\
3 \mathrm{LA} \\
4 \mathrm{LA}\end{array}$ & $\mathrm{ChBr}$ & $\begin{array}{l}0.665 \\
0.654 \\
0.675\end{array}$ & $\begin{array}{l}0.032 \\
0.022 \\
0.049\end{array}$ & $\begin{array}{l}1.308 \\
1.293 \\
1.318\end{array}$ & $\begin{array}{c}0.162 \\
0.430 \\
0.390^{c}\end{array}$ \\
\hline $\begin{array}{c}\text { Folk et at. } \\
\text { 1999, Ref. }\end{array}$ & $\begin{array}{c}\text { MS, 3d } \\
\text { mass }\end{array}$ & $\begin{array}{l}4 \mathrm{LA} \\
4 \mathrm{LA}\end{array}$ & $\begin{array}{l}\mathrm{ChBr} \\
\mathrm{ChBr}\end{array}$ & & & & $\begin{array}{l}0.39(4)^{c} \\
0.372(5)\end{array}$ \\
\hline 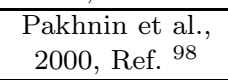 & mass & $5 \mathrm{LA}$ & $\mathrm{PdBr}$ & $0.671(5)$ & $0.025(10)$ & $1.325(3)$ & $0.32(6)$ \\
\hline $\begin{array}{l}\text { Varnashevis } \\
2000 \text { Ref. }\end{array}$ & mass & $4 \mathrm{LA}$ & $\begin{array}{l}\mathrm{PdBr} \\
\mathrm{PdBr}\end{array}$ & $\begin{array}{c}0.681(12) \\
0.672(4)\end{array}$ & $\begin{array}{l}0.040(11) \\
0.034(10)\end{array}$ & $\begin{array}{l}1.336(20) \\
1.323(10)\end{array}$ & 0.330 \\
\hline $\begin{array}{l}\text { Pelissetto et al., } \\
\text { 2000, Ref. } 118\end{array}$ & mass & $6 \mathrm{LA}$ & $\begin{array}{l}\mathrm{PdBr}- \\
\mathrm{CM} \\
\mathrm{PdBr}- \\
\mathrm{PdBr}\end{array}$ & $\begin{array}{l}0.678(10) \\
0.668(6)\end{array}$ & $\begin{array}{c}0.030(3) \\
0.0327(19)\end{array}$ & $\begin{array}{l}1.330(17) \\
1.313(14)\end{array}$ & $\begin{array}{l}0.25(10) \\
0.25(10)\end{array}$ \\
\hline $\begin{array}{l}\text { Tissier et } 2 \mathrm{l} \cdot \mathrm{r} \\
\text { 2001, Ref. }\end{array}$ & EEA & No & & 0.67 & 0.05 & 1.306 & \\
\hline
\end{tabular}

\subsection{Systems with long-range-correlated disorder}

\subsubsection{Magnets with long-range correlated disorder}

Now, let us discuss the results obtained for the $m$-vector model with long-rangecorrelated disorder described by the effective Hamiltonian (25). A one-loop approximation was given in Ref. 4 using an expansion in $\varepsilon=4-d, \delta=4-a$. A new long-range-correlated fixed point was found with a correlation-length exponent $\nu=2 / a$ and it was argued, that this new scaling relation is exact and also holds in 
higher order approximations. This result was questioned recently in Refs. 00 , where the static and dynamic properties of $3 \mathrm{~d}$ systems with long-range-correlated disorder were studied by means of the massive field-theoretical RG approach in a 2-loop approximation, for different fixed values of the correlation parameter, $2 \leq a \leq 3$. The $\beta$ and $\gamma$ functions in the two-loop approximation were calculated as an expansion series in renormalized vertices $u, v$ and $w$. The Padé-Borel resummation was used for their analysis. The study revealed the existence of the stable random fixed point with $u^{*} \neq 0, v^{*} \neq 0, w^{*} \neq 0$ in the whole region of the parameter $a$. The comparison of the obtained exponents $\nu$ values at various $a$ and ratio $2 / a$ shows the violation of the supposed exact the relation $\nu=2 / a$ of ref.44. The authors concluded that the revealed difference is caused by the use of a more accurate field-theoretical description using higher order approximations for the $3 \mathrm{~d}$ system directly together with methods of series summation. Recently, the correction-to-scaling exponent for magnets with long-range-correlated defects was obtained using the same approach 69.

\subsubsection{Polymers in media with long-range-correlated quenched disorder}

The effective Hamiltonian (26) is the starting point to study the polymer limit $m \rightarrow 0$ of the weakly diluted Stanley model with long-range-correlated disorder. As we explained in the subsection 3.6 it may serve as a model for polymers in porous media. Again, imposing the renormalization conditions of the massive scheme (32), (33) the one-loop approximation leads to the following expressions for the $\mathrm{RG}$ functions 70 :

$$
\begin{gathered}
\beta_{u}=-\varepsilon\left[u-\frac{4}{3} u^{2} I_{1}\right]-\delta 2 u w\left[I_{2}+\frac{1}{3} I_{4}\right]+(2 \delta-\varepsilon) \frac{2}{3} w^{2} I_{3}, \\
\beta_{w}=-\delta\left[w+\frac{2}{3} w^{2} I_{2}\right]+\varepsilon \frac{2}{3}\left[w u I_{1}-w^{2} I_{4}\right], \\
\bar{\gamma}_{\phi^{2}}=\varepsilon \frac{u}{3} I_{1}-\delta \frac{w}{3} I_{2}, \quad \gamma_{\phi}=\delta \frac{w}{3} I_{4} .
\end{gathered}
$$

Here, $I_{i}$ are one-loop integrals that depend on the space dimension $d$ and the correlation parameter $a$ :

$$
\begin{aligned}
I_{1}=\int \frac{\mathrm{d} \vec{q}}{\left(q^{2}+1\right)^{2}}, \quad I_{2} & =\int \frac{\mathrm{d} \vec{q} q^{a-d}}{\left(q^{2}+1\right)^{2}}, \quad I_{3}=\int \frac{\mathrm{d} \vec{q} q^{2(a-d)}}{\left(q^{2}+1\right)^{2}}, \\
I_{4} & =\frac{\partial}{\partial k^{2}}\left[\int \frac{\mathrm{d} \vec{q} q^{a-d}}{[q+k]^{2}+1}\right]_{k^{2}=0} .
\end{aligned}
$$

Note that in contrast to the usual $\phi^{4}$ theory the $\gamma_{\phi}$ function in Eq. 63) is nonzero already at the one-loop level. This is due to the $k$-dependence of the integral $I_{4}$ in Eq. 64).

There are essentially two ways to proceed in order to obtain the qualitative characteristics of the critical behavior of the model. The first is to consider the 
polynomials in Eqs. (61), (62) for fixed $a, d$ and look for the solution of the fixed point equations. It is easy to check that these one-loop equations do not have any stable accessible fixed points for $d<4$. The second scheme is to evaluate these equations in a double expansion in $\varepsilon=4-d$ and $\delta=4-a$ as proposed by Weinrib and Halperin 14. Substituting the loop integrals in Eqs. (61)-(63) by their expansion in $\varepsilon=4-d$ and $\delta=4-a$, one obtains the 3 fixed points given in the table 4 . The

Table 4. Fixed points and stability matrix eigenvalues in the first order of the $\varepsilon, \delta$ - expansion 80

\begin{tabular}{|c|cccc|}
\hline Fixed Point & $u^{*}$ & $w^{*}$ & $\omega_{1}$ & $\omega_{2}$ \\
\hline Gaussian $(\mathbf{G})$ & 0 & 0 & $-\varepsilon$ & $-\delta$ \\
\hline Pure SAW $(\mathbf{P})$ & $\varepsilon$ & 0 & $\varepsilon$ & $\varepsilon / 2-\delta$ \\
\hline Long-range $(\mathbf{L R})$ & $\frac{2 \delta^{2}}{(\varepsilon-\delta)}$ & $-\frac{\delta(\varepsilon-2 \delta)}{(\varepsilon-\delta)}$ & $\frac{1}{2}\left\{\varepsilon-4 \delta \pm \sqrt{\varepsilon^{2}-4 \varepsilon \delta+8 \delta^{2}}\right\}$ & \\
\hline
\end{tabular}

following conclusions may be drawn from these first order results: Three distinct accessible fixed points are found to be stable in different regions of the $a, d$-plane: the Gaussian (G) fixed point, the pure (P) SAW fixed point and the long-range $(\mathbf{L R})$ disorder SAW fixed point. The corresponding regions in the $a, d$-plane are marked by I, II and III in Fig. 9. In the region IV no stable fixed point is accessible.

For the correlation length critical exponent of the SAW, one finds distinct values $\nu_{\text {pure }}$ for the pure fixed point and $\nu_{\mathrm{LR}}$ for the long-range fixed point. Taking into account that the accessible values of the couplings are $u>0, w>0$, one finds that the long-range stable fixed point is accessible only for $\delta<\varepsilon<2 \delta$, or $d<a<2+d / 2$, a region where power counting in Eq. (26) shows that the disorder is irrelevant. In this sense the region III for the stability of the LR fixed point is unphysical. Formally, the first order results for $d<4$ read:

$$
\nu= \begin{cases}\nu_{\text {pure }}=1 / 2+\varepsilon / 16, & \delta<\varepsilon / 2, \\ \nu_{\mathrm{LR}}=1 / 2+\delta / 8, & \varepsilon / 2<\delta<\varepsilon .\end{cases}
$$

Thus, in this linear approximation the asymptotic behavior of polymers is governed by a distinct exponent $\nu_{\mathrm{LR}}$ in the region III of the parameter plane $a, d$. However, the region where the $\mathbf{L R}$ fixed point is found appears to be unphysical.

Something similar happens if the $\varepsilon, \delta$-expansion is applied to study models of $m$-vector magnets with long-range-correlated quenched disorder 14,69: also in the case of magnets, as well as for polymers the first order $\varepsilon, \delta$-expansion leads to a controversial phase diagram. In order to obtain a clear picture and more reliable information, one should proceed to higher order calculations.

To investigate the peculiarities of the critical behavior in the 2-loop approximation one may make use of the $m \rightarrow 0$ limit of the appropriate $m$-vector model (25), investigated recently 80 . Starting from the two-loop expressions of Ref. 30 for the RG functions of the Stanley model with long-range-correlated disorder and making use of the symmetry arguments 62 in the polymer limit $m=0$ one gets the following expressions for the $d=3 \mathrm{RG}$ functions of the model described by the effective Hamiltonian (26) 6 : 


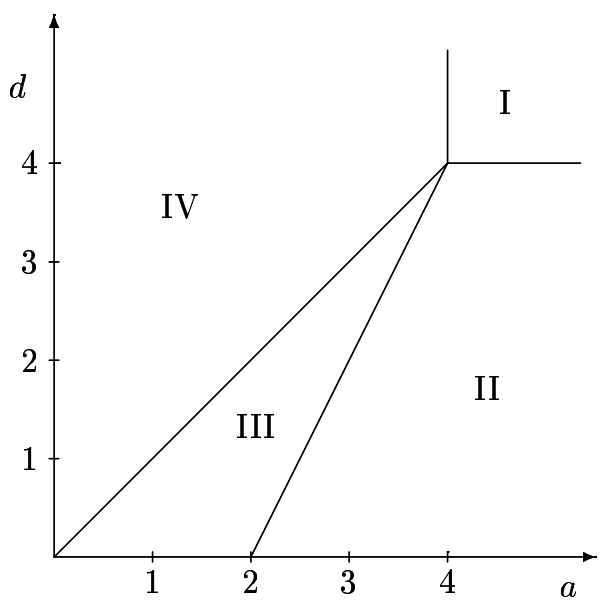

Fig. 9. The critical behavior of a polymer in a medium with long-range-correlat disorder in different regions of the $d, a$-plane as predicted by the first order $\varepsilon, \delta$-expansion 70 . Region I corresponds to the Gaussian random walk behavior, in the region II scaling behavior is the same as in the medium without disorder, in region III the "long-range" fixed point LR is stable and the scaling laws for polymers are altered, in region IV no accessible stable fixed points appear; this may be interpreted as the collapse of the chain.

$$
\begin{gathered}
\beta_{u}(u, w)=-u+u^{2}-\left(3 f_{1}(a)-f_{2}(a)\right) u w-\frac{95}{216} u^{3}+\frac{1}{8} b_{2}(a) u^{2} w- \\
\left(b_{3}(a)-\frac{1}{4} b_{6}(a)\right) u w^{2}+f_{3}(a) w^{2}+b_{5}(a) w^{3}, \\
\beta_{w}(u, w)=-(4-a) w-\left(f_{1}(a)-f_{2}(a)\right) w^{2}+\frac{u w}{2}+b_{10}(a) w^{3}- \\
\frac{23}{216} u^{2} w+\frac{1}{4} b_{12}(a) u w^{2}, \\
\gamma_{\phi}(u, w)=\frac{1}{2} f_{2}(a) w+\frac{1}{108} u^{2}+c_{1}(a) w^{2}-\frac{1}{4} c_{2}(a) u w, \\
\bar{\gamma}_{\phi^{2}}(u, w)=\frac{1}{4} u-\frac{1}{2} f_{1}(a) w-\frac{1}{16} u^{2}-c_{3}(a) w^{2}+\frac{1}{4} c_{4}(a) u w .
\end{gathered}
$$

Here, the coefficients $f_{i}(a)$ are expressed in terms of the one-loop integrals in Eq. (64), $b_{i}(a)$ and $c_{i}(a)$ originate from the two-loop integrals and are tabulated in Ref. 80 for $d=3$ and different values of the parameter $a$ in the range $2 \leq a \leq 3$. The series are normalized by a standard change of variables $u \rightarrow \frac{3 u}{4} I_{1}, w \rightarrow \frac{w}{32} I_{1}$, so that the coefficients of the terms $u, u^{2}$ in $\beta_{u}$ become 1 in modulus.

As in the former cases discussed in subsections 6.1, 6.2.1 the question about the summability of the series in Eqs. (66) - (69) is open. In Ref. 69 various kinds of resummation techniques have been applied in order to obtain reliable quantitative results for the system under consideration and to check the stability of these results. 


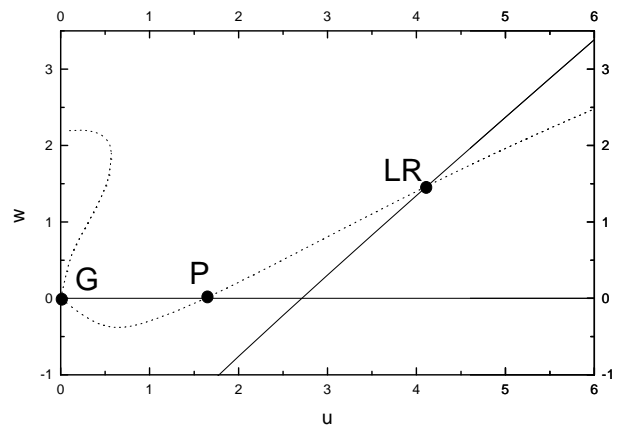

Fig. 10. The lines of zeroes of the $3 \mathrm{~d} \beta$-functions (66), 67 resummed by the Chisholm-Borel method at $a=2.9$. The dashed line corresponds to $\beta_{u}=0$, the solid lines depict $\beta_{w}=0$. The intersections of the dashed and solid lines give three fixed points shown by filled circles at $u^{*}=0, w^{*}=0(\mathbf{G}), u^{*}=1.63, w^{*}=0(\mathbf{P})$, and $u^{*}=4.13, w^{*}=1.47(\mathbf{L R})$. The fixed point $\mathbf{L R}$ is stable.

First, a simple two-variable Chisholm-Borel resummation technique was employed, which turns out to be the most effective one for the given problem. In addition to the familiar fixed points $\mathbf{G}$ and $\mathbf{P}$ describing Gaussian chains and polymers (SAWs) on regular lattices, the stable long-range fixed point $\mathbf{L R}$ for polymers in long-rangecorrelated disorder was found. In Fig. 10 the lines of zeroes of the resummed $\beta$ functions (66), (67) at $a=2.9$ in the $u, w$-plane in the region of interest are depicted. The intersections of these curves correspond to the fixed points. The corresponding values of the stable fixed point coordinates and the stability matrix eigenvalues for different values of the correlation parameter $a<3$ are given in Table 5 .

To calculate the critical exponents the same resummation technique was applied. The numerical values for $\nu, \gamma$ and $\eta$ are listed in Table 0 for $a=2.3, \ldots, 2.9$. Note, that for $a=3$, which corresponds to short-range-correlated point-like defects, the interactions $u$ and $w$ become of the same symmetry, so one can pass to one coupling $(u-w)$ and reproduce the well-known values of the critical exponents for the pure SAW model. The numerical values corresponding to those listed in Table 5 in this case read: $u^{*}=1.63, \nu=0.59, \gamma=1.17, \eta=0.02, \omega=0.64$. As departing from the value $a=3$ downward to 2 one notices a major increase of the value of the coupling $u$, so the results are more reliable for $a$ close to 3 . At some value $a=a_{\text {marg }}$ the $\mathbf{L R}$ fixed point becomes unstable.

To verify the results, the method of subsequent resummation (see subsection 5.4 was applied to the RG functions (66), 67). Here, the summation was carried out first in the coupling $u$ and subsequently in $w$. Again, the presence of stable fixed point $\mathbf{L R}$ for $a_{\text {marg }} \leq a<d$ was confirmed.

The obtained results may be summarized and interpreted as follows: 
Table 5. Stable fixed point of the $3 \mathrm{~d} 2$-loop $\beta$-functions, resummed by Chisholm-Borel method, the corresponding critical exponents and the stability matrix eigenvalues at various values of $a$.

\begin{tabular}{|c|c|c|c|c|c|c|}
\hline$a$ & $u^{*}$ & $w^{*}$ & $\nu$ & $\gamma$ & $\eta$ & $\omega_{1,2}$ \\
\hline 2.9 & 4.13 & 1.47 & 0.64 & 1.25 & 0.04 & $0.25 \pm 0.62 \mathrm{i}$ \\
2.8 & 4.73 & 1.68 & 0.64 & 1.26 & 0.04 & $0.22 \pm 0.76 \mathrm{i}$ \\
2.7 & 5.31 & 1.81 & 0.65 & 1.28 & 0.03 & $0.18 \pm 0.89 \mathrm{i}$ \\
2.6 & 5.89 & 1.87 & 0.66 & 1.29 & 0.03 & $0.15 \pm 0.99 \mathrm{i}$ \\
2.5 & 6.48 & 1.89 & 0.66 & 1.31 & 0.02 & $0.11 \pm 1.09 \mathrm{i}$ \\
2.4 & 7.10 & 1.87 & 0.67 & 1.33 & 0.01 & $0.07 \pm 1.18 \mathrm{i}$ \\
2.3 & 7.76 & 1.84 & 0.68 & 1.36 & 0.01 & $0.03 \pm 1.26 \mathrm{i}$ \\
\hline
\end{tabular}

(i) A new stable fixed point (LR) for polymers in long-range-correlated disorder is found for $d=3, a<d$, leading to critical exponents that are different from those of the pure model;

(ii) There is a marginal value $a_{\text {marg }}$ for the parameter $a$, below which the stable fixed point is absent, indicating a chain collapse of the polymer for disorder that is stronger correlated.

(iii) The critical exponent $\nu$ increases with decreasing parameter $a$, like in the Weinrib and Halperin case. But the relation $\nu=2 / a$ does not hold. Physically this means that in weak long-range-correlated disorder $\left(a>a_{\text {marg }}\right)$ the polymer coil swells with increasing correlation of the disorder. The self avoiding path of the polymer has to take larger deviations to avoid the defects of the medium.

\subsection{Critical behaviour of Stanley model with random anisotropy}

The RG results discussed so far in the subsections 6.1, 6.2 concerned randomtemperature like disorder. Another type of disorder that we consider here is the disorder induced by a single-ion anisotropy term in the RAM Hamiltonian (13). According to the chosen distributions of the anisotropy axis (14) and (15) our consideration falls into two parts. First we analyze the case of the RAM with isotropic distributions of anisotropy axis and then we discuss what peculiarities in the critical behavior of the RAM might appear if the random-anisotropy axis is taken to point only along the edges of $m$-dimensional hypercube (we call this the "cubic" distribution).

\subsubsection{An isotropic distribution of the random anisotropy axis}

Applying the renormalization conditions of the massive scheme (32) to the effective Hamiltonian (29) one obtains the two-loop RG functions, that in the replica limit $n=0$ read $124:$

$$
\begin{aligned}
\beta_{u}= & -(4-d) u\left\{1-\frac{1}{6}[(m+8) u+12 v+2(m+5) w]+\frac{1}{9}\left[2(5 m+22) u^{2}+84 v^{2}+\right.\right. \\
& \left.24(m+5) u v+2(14 m+58) w u+4(9 m+33) v w+(17 m+67) w^{2}\right] i_{1}+\frac{2}{9}\left[2 v^{2}+\right.
\end{aligned}
$$




$$
\begin{aligned}
& \left.\left.2(m+2) u v+(m+2) u^{2}+\frac{m+3}{2} w^{2}+2(m+2) u w+2(m+1) v w\right] i_{2}\right\} \\
\beta_{v}= & (4-d)\left\{v-\frac{1}{6}\left[8 v^{2}+2(m+2) u v+2 u w+2(m+1) v w+3 w^{2}\right]+\frac{1}{9}\left[44 v^{3}+\right.\right. \\
& 24(m+2) u v^{2}+2(3 m+6) v u^{2}+2(6 m+24) u v w+2(12 m+12) w v^{2}+ \\
& \left.(3 m+45) v w^{2}+2(m+8) u w^{2}+4 u^{2} w+(3 m+9) w^{3}\right] i_{1}+\frac{2}{9}\left[2 v^{3}+(m+2) v u^{2}+\right. \\
2 & \left.\left.(m+2) u v^{2}+\frac{m+3}{2} w^{2} v+2(m+2) u v w+2(m+1) v^{2} w\right] i_{2}\right\} \\
\beta_{w}= & -(4-d) w\left\{1-\frac{1}{6}[(m+4) w+12 v+4 u]+\frac{1}{9}\left[(5 m+27) w^{2}+84 v^{2}+\right.\right. \\
& \left.4(6 m+15) v w+2(5 m+22) u w+4(3 m+18) u v+2(m+6) u^{2}\right] i_{1}+\frac{2}{9}\left[2 v^{2}+\right. \\
& \left.\left.2(m+2) u v+(m+2) u^{2}+\frac{m+3}{2} w^{2}+2(m+2) u w+2(m+1) v w\right] i_{2}\right\} \\
\gamma_{\phi}= & \frac{(4-d)}{9}\left(2 v^{2}+2(m+2) u v+(m+2) u^{2}+2(m+2) u w+\frac{m+3}{2} w^{2}+\right. \\
& 2(m+1) v w) i_{2}, \\
\bar{\gamma}_{\phi^{2}}= & \frac{(4-d)}{3}\left\{\frac{1}{2}(2 v+(m+2) u+(m+1) w)-\left(2 v^{2}+2(m+2) u v+\right.\right. \\
& \left.\left.(m+2) u^{2}+2(m+2) u w+\frac{m+3}{2} w^{2}+2(m+1) v w\right) i_{1}\right\}
\end{aligned}
$$

Here, $u, v, w$ are the renormalized couplings, $i_{1}, i_{2}$ are the two-loop integrals (c. f. formulas (52), (53) and comments below them).

Again, in a first step of the analysis one may use the $\varepsilon$-expansion. The first order $\varepsilon$-expansion results were given in Ref. $\mathrm{W}$. They can be reproduced from formulas (70) - (72). In particular one gets eight fixed points with the coordinates given in Table 6 125. The second order contributions in $\varepsilon$ to the fixed point coordinates calculated in Ref. 124 do not change qualitatively the results obtained 126. The main question of interest now is whether the above described picture of the runaway solution is not an artifact of an $\varepsilon$-expansion. To shed light on this question one can use a more refined analysis of the fixed points and their stability, similar as it was done in the subsections 6.1, 6.2.

All fixed points with $u>0, v>0, w<0$ appear to be unstable for $\varepsilon>0$ except of the "polymer" $O(n=0)$ fixed point III which is stable for all $m$. However, the presence of a stable fixed point is not a sufficient condition for the 2 nd order phase 
Table 6. Fixed points of the RAM with isotropic distribution of the random anisotropy axis in $\varepsilon$-expansion. Here, $x_{ \pm}=\left(m-2 \pm \sqrt{(m-2)^{2}+48}\right) / 8, y_{ \pm}=(m-2-2 m z \pm$ $\left.\sqrt{(m-2-2 m z)^{2}+4(12-8 z)}\right) / 8, z=(m+6) /(m+8)$.

\begin{tabular}{|l|c|c|c|}
\hline & $u^{*}$ & $v^{*}$ & $w^{*}$ \\
\hline I. & 0 & 0 & 0 \\
II. & $\frac{6}{m+8} \varepsilon$ & 0 & 0 \\
III. & 0 & $\frac{6}{8} \varepsilon$ & 0 \\
IV. & $\frac{3}{2(m-1)} \varepsilon$ & $-\frac{3(4-m)}{8(m-1)} \varepsilon$ & 0 \\
V. & 0 & $\frac{6 x_{+}}{12 x_{+}+m+4} \varepsilon$ & $\frac{6}{12 x_{+}+m+4} \varepsilon$ \\
VI. & 0 & $\frac{6 x_{-}}{12 x_{-}+m+4} \varepsilon$ & $\frac{6}{6 y_{+}} \varepsilon$ \\
VII. & $-\frac{6 z}{12 y_{+}-4 z+m+4} \varepsilon$ & $\frac{6}{12 y_{+}-4 z+m+4} \varepsilon$ & $\frac{6 y_{-}}{12 y_{+}-4 z+m+4} \varepsilon$ \\
VIII. & $-\frac{6 z}{12 y_{-}-4 z+m+4} \varepsilon$ & $\frac{6}{12 y_{-}-4 z+m+4} \varepsilon$ & $\frac{6}{12 y_{-}-4 z+m+4} \varepsilon$ \\
\hline
\end{tabular}

transition. The fixed point should be accessible from the initial values of couplings and it is not the case for the location of fixed points shown in Fig. 11. Recall that the initial conditions of the RAM effective Hamiltonian in our case are given by $u_{0}>0, v_{0}>0, w_{0}<0$ and $w_{0} / v_{0}=-m$ (see subsection 4.3). Indeed, starting from the region of initial conditions (denoted by a cross in the figure) for zero value of $u$ one meets a separatrix joining the unstable fixed points I and VI and the system cannot reach the stable fixed point III. As far as both fixed points I and VI are strongly unstable with respect to $u$, the fixed point III is not accessible for arbitrary positive $u$ either. Finally one concludes that the 2 nd order phase transition is absent in the model as shown by runaway solutions of the RG equations.

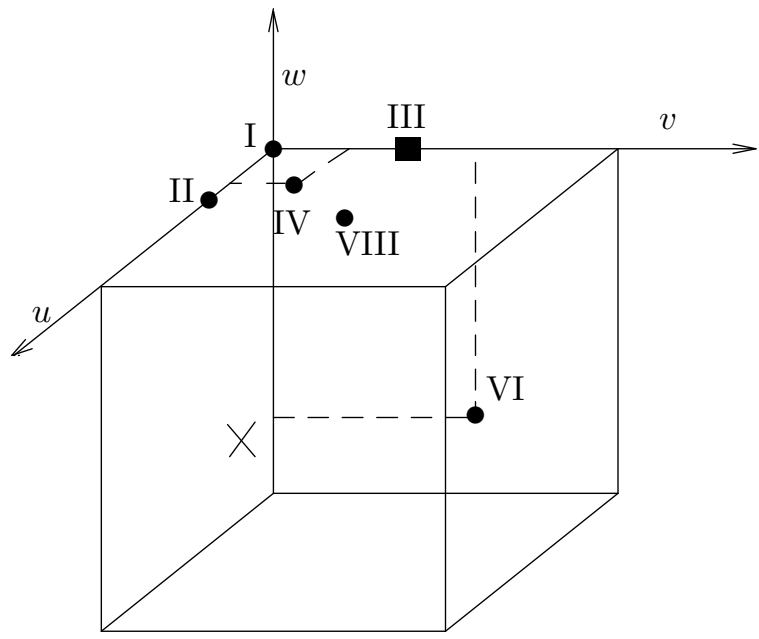

Fig. 11. Fixed points of the RAM with isotropic distribution of a local anisotropy axis. The only fixed points located in the octant $u>0, v>0, w<0$ are shown. The filled box shows the stable fixed point, the cross denotes typical initial values of the couplings.

Considering the two-loop RG functions (70)-(72) directly at $d=3$ and applying 
the Padé-Borel resummation (45)-(47) one gets 124,227 the fixed point coordinates given in the Table 0126. Resummed two-loop results qualitatively confirm the picture obtained in the first order in $\varepsilon$-expansion: the stability of the fixed points does not change after resummation. This supports a conjecture of Aharony 19 about the absence of an accessible stable fixed point for the RAM with isotropic distribution of the local anisotropy axis.

In the other fixed points one may recover the two-loop results for the $O(m)$ model (fixed point II), the polymer $O(n=0)$ model (III) and the diluted Stanley model (IV). The fixed point VIII contains all three couplings but is both unstable and not accessible from the initial values of couplings. The values of the correlation length and pair correlation function critical exponents $\nu$ and $\eta$, resummed in a similar way, are given in the table. When calculated in unstable fixed points, they are considered as effective exponents.

Table 7. Resummed values of the fixed points and critical exponents for isotropic case in two-loop approximation for $d=322,126$.

\begin{tabular}{|c|c|c|c|c|c|c|}
\hline FP & $m$ & $u^{*}$ & $v^{*}$ & $w^{*}$ & $\nu$ & $\eta$ \\
\hline I & $\forall m$ & 0 & 0 & 0 & & \\
\hline II & 2 & 0.9107 & 0 & 0 & 0.663 & 0.027 \\
& 3 & 0.8102 & 0 & 0 & 0.693 & 0.027 \\
& 4 & 0.7275 & 0 & 0 & 0.724 & 0.027 \\
\hline III & $\forall m$ & 0 & 1.1857 & 0 & 0.590 & 0.023 \\
\hline IV & 2 & 0.9454 & -0.0322 & 0 & 0.668 & 0.027 \\
& 3 & 0.6460 & 0.1733 & 0 & 0.659 & 0.027 \\
& 4 & 0.4851 & 0.2867 & 0 & 0.653 & 0.028 \\
\hline VI & 2 & 0 & 1.4650 & -1.6278 & 0.449 & -0.028 \\
\hline VIII & 2 & 0.7991 & 0.7311 & -0.5360 & 0.618 & 0.026 \\
& 3 & 0.8450 & 0.5934 & -0.4506 & 0.614 & 0.025 \\
& 4 & 0.8762 & 0.5060 & -0.3956 & 0.612 & 0.025 \\
\hline
\end{tabular}

\subsubsection{A cubic distribution of the random anisotropy axis}

Now we turn our attention to the case of the RAM with a cubic distribution of the random anisotropy axis (15). The RG functions that correspond to the effective Hamiltonian (29) in two-loop approximation were obtained in Ref. 128 within the massive renormalization scheme (32). In the replica limit $n=0$ they read:

$$
\begin{aligned}
\beta_{u}= & -\varepsilon u\left\{1-\frac{1}{6}[(m+8) u+12 v+4 w+6 y]+\frac{1}{9}\left[2(5 m+22) u^{2}+8(3 m+15) v u+\right.\right. \\
& \left.84 v^{2}+12 w^{2}+68 u w+72 u y+18 y^{2}+72 v w+108 v y+36 w y\right] i_{1}+\frac{2}{9}\left[(m+2) u^{2}+\right. \\
& \left.\left.2(m+2) u v+2 v^{2}+2 w^{2}+6 u w+4 v w+6 v y+6 u y+6 w y+3 y^{2}\right] i_{2}\right\}
\end{aligned}
$$




$$
\begin{aligned}
\beta_{v}= & -\varepsilon\left\{v-\frac{1}{6}\left[8 v^{2}+2(m+2) u v+2 u w+4 v w+6 v y\right]+\frac{1}{9}\left[44 v^{3}+48 v^{2} w+\right.\right. \\
& 12 w^{2} v+24(m+2) u v^{2}+2(3 m+6) v u^{2}+4 w^{2} u+4 u^{2} w+72 v^{2} y+18 y^{2} v+ \\
& 60 u v w+36 u v y+36 v w y] i_{1}+\frac{2}{9}\left[2 v^{3}+2(m+2) u v^{2}+(m+2) v u^{2}+\right. \\
& \left.\left.2 w^{2} v+6 u v w+4 v^{2} w+6 v^{2} y+6 u v y+6 v w y+3 y^{2} v\right] i_{2}\right\} \\
\beta_{w}= & -\varepsilon w\left\{1-\frac{1}{6}[8 w+12 v+4 u+6 y]+\frac{1}{9}\left[44 w^{2}+84 v^{2}+120 w v+2(m+6) u^{2}+\right.\right. \\
& \left.68 u w+72 w y+18 y^{2}+2(6 m+36) u v+108 v y+36 u y\right] i_{1}+\frac{2}{9}\left[(m+2) u^{2}+\right. \\
& \left.\left.2(m+2) u v+2 v^{2}+2 w^{2}+6 u w+4 v w+6 v y+6 u y+6 w y+3 y^{2}\right] i_{2}\right\} \\
\beta_{y}= & -\varepsilon\left\{y-\frac{1}{6}\left[9 y^{2}+8 u w+12 v y+12 u y+12 w y\right]+\frac{1}{9}\left[(4 m+72) u^{2} w+72 w^{2} u+\right.\right. \\
& 54 y^{3}+84 v^{2} y+(6 m+84) u^{2} y+84 w^{2} y+144 y^{2} v+144 y^{2} u+144 y^{2} w+ \\
& 96 u v w+2(6 m+84) u v y+252 u w y+168 v w y] i_{1}+\frac{2}{9}\left[2 v^{2} y+2(m+2) u v y+\right. \\
\bar{\gamma}_{\phi^{2}}= & \left(m\left\{\frac{1}{3}\left(2 v+(m+2) u+2 w+2 w^{2} y+6 u w y+4 v w y+6 y^{2} v+6 y^{2} u+6 y^{2} w+3 y^{3}\right] i_{2}\right\}\right. \\
& \left.\left.6 u w+2 w^{2}+4 v w+6 v y+6 u y+6 w y+3 y^{2}\right) i_{1}\right\} \\
\gamma_{\phi}=- & \frac{\varepsilon}{9}\left(2 v^{2}+2(m+2) u v+(m+2) u^{2}+6 u w+2 w^{2}+4 v w+6 v y+\right. \\
& \left.6 u y+6 w y+3 y^{2}\right) i_{2}, \\
&
\end{aligned}
$$

Again, $i_{1}, i_{2}$ stand for the two-loop integrals and the renormalized couplings $u, v$, $w$ and $y$ correspond to the effective Hamiltonian (29).

As in the isotropic case the first RG result for the RAM with cubic distribution of local anisotropy axis was obtained in the first order in $\varepsilon$ 19. It brought about the evidence of 14 fixed points and may be easily reproduced from the functions (75)-(80) removing the two-loop contributions. The coordinates of the fixed points are listed in the Table 825 .

The results of the linear $\varepsilon$ analysis 19 states that among the fixed points with $u>0, v>0, w<0$ only a "polymer" $O(n=0)$ fixed point III is stable for all $m$ for $\varepsilon>0$, but it is not reachable from the initial values of couplings (see Fig. 12). The 
Table 8. Fixed points of the RAM with the random cubic anisotropy distribution ( $\varepsilon$-expansion). Here, $\alpha_{ \pm}=\left(m-4 \pm \sqrt{m^{2}+48}\right) / 8, \beta_{ \pm}=-\left(m+12 \pm \sqrt{m^{2}+48}\right) / 6, A_{ \pm \pm}=6 \alpha_{ \pm}+3 \beta_{ \pm}+m+6$. The fixed points XV-XVII appear only in the two-loop approximation due to the degeneracy of the corresponding one-loop functions.

\begin{tabular}{|l|c|c|c|c|}
\hline & $u^{*}$ & $v^{*}$ & $w^{*}$ & $y^{*}$ \\
\hline I. & 0 & 0 & 0 & 0 \\
II. & $\frac{6}{m+8} \varepsilon$ & 0 & 0 & 0 \\
III. & 0 & $\frac{6}{8} \varepsilon$ & 0 & 0 \\
IV. & 0 & 0 & $\frac{6}{8} \varepsilon$ & 0 \\
V. & 0 & 0 & 0 & $\frac{6}{9} \varepsilon$ \\
VI. & 6 & $\frac{6(m-4)}{4(m-1)} \varepsilon$ & 0 & 0 \\
VII. & 0 & $\frac{3}{2} \varepsilon$ & $-\frac{3}{2} \varepsilon$ & 0 \\
VIII. & $\frac{2}{m} \varepsilon$ & 0 & 0 & $\frac{2(m-4)}{3 m} \varepsilon$ \\
IX. $m \neq 2$ & $\frac{1}{m-2} \varepsilon$ & $\frac{m-4}{4(m-2)} \varepsilon$ & 0 & $\frac{m-4}{3(m-2)} \varepsilon$ \\
X. & 0 & $\frac{1}{2} \varepsilon$ & $-\frac{1}{2} \varepsilon$ & $\frac{2}{3} \varepsilon$ \\
XI. & $\frac{3}{A_{++}} \varepsilon$ & $\frac{3 \alpha_{+}}{A_{++}} \varepsilon$ & $\frac{3(m+4)}{4 A_{++}} \varepsilon$ & $\frac{3 \beta_{+}}{A_{+}+} \varepsilon$ \\
XII. & $\frac{3}{A_{+-}} \varepsilon$ & $\frac{3 \alpha_{+}}{A_{+-}} \varepsilon$ & $\frac{3(m+4)}{4 A_{+-}} \varepsilon$ & $\frac{3 \beta_{-}}{A_{+-}} \varepsilon$ \\
XIII. & $\frac{3}{A_{-+}} \varepsilon$ & $\frac{3 \alpha_{-}}{A_{-+}} \varepsilon$ & $\frac{3(m+4)}{4 A_{-+}} \varepsilon$ & $\frac{3 \beta_{+}}{A_{-}+} \varepsilon$ \\
XIV. & $\frac{3}{A_{--}} \varepsilon$ & $\frac{3 \alpha_{-}}{A_{--}} \varepsilon$ & $\frac{3(m+4)}{4 A_{--}} \varepsilon$ & $\frac{3 \beta_{-}}{A_{--}} \varepsilon$ \\
\hline XV. & 0 & 0 & $\mp \sqrt{\frac{54}{53}} \varepsilon$ & $\pm \frac{4}{3} \sqrt{\frac{54}{53}} \varepsilon$ \\
XVI. & 0 & $\mp \sqrt{\frac{54}{53}}$ & 0 & $\pm \frac{4}{3} \sqrt{\frac{54}{53} \varepsilon}$ \\
XVII. $m=2$ & $\mp 2 \sqrt{\frac{54}{53} \varepsilon}$ & $\pm \sqrt{\frac{54}{53} \varepsilon}$ & 0 & $\pm \frac{4}{3} \sqrt{\frac{54}{53} \varepsilon}$ \\
\hline
\end{tabular}

reason is a separatrix joining the unstable fixed points I and VII and separating initial values of couplings (shown by a cross in the Fig. 12) and fixed point III. The possible runaway behavior of the RG flow lead to the conclusion about a smearing out of the phase transition as $T_{c}$ is approached 19 . However, a subsequent study of Mukamel and Grinstein 129 brought about the possibility of a second order phase transition with the scenario of a weakly diluted quenched Ising model 76 . Indeed, accounting for the $\varepsilon^{2}$ terms qualitatively changes the picture: performing the perturbation theory expansion one gets 126,126 not only the corrections to the coordinates of the fixed points I-XIV (we do not display them here as far as they as are too cumbersome) but also the new fixed points XV, XVI, XVII (see the bottom of the Table 8). The appearance of the pairs of fixed points denoted by XV and $\mathrm{XVI}$ is caused by the fact that the $\beta$-functions $\beta_{w}, \beta_{y}$ at $u=v=0\left(\beta_{u}, \beta_{v}\right.$ at $w=y=0$, correspondingly) are degenerated at the one loop level. This leads to the $\sqrt{\varepsilon}$-expansion (c.f. subsection 6.1) of the weakly diluted quenched Ising model. The $\sqrt{\varepsilon}$ expansion of the fixed point XVII holds only for $m=2$ and is caused by the one-loop degeneracy of the $\beta_{u}, \beta_{v}, \beta_{y}$ functions for $w=0$ (c. f. singularity at $m=2$ in the $\varepsilon$-expansion of the fixed point IX) 128 .

Checking the stability of the new fixed points one finds that all of them are unstable except the Ising-like fixed point with $w<0, y>0$ of the pair XV. Moreover, this point is reachable from the initial values of the couplings. As far as this is the fixed point of the diluted Ising model one concludes, that in the critical region the RAM with cubic distribution of the random anisotropy axis (15) decouples into $m$ 
independent dilute Ising models and the phase transition for any $m$ is governed by the familiar random Ising model critical exponents. However, it is worthwhile to keep in mind that above picture is obtained in the frames of the "naive" analysis of the $\varepsilon$ (and $\sqrt{\varepsilon}$ ) expansion and it is highly desirable to confirm it by a more reliable analysis of fixed points and their stability.

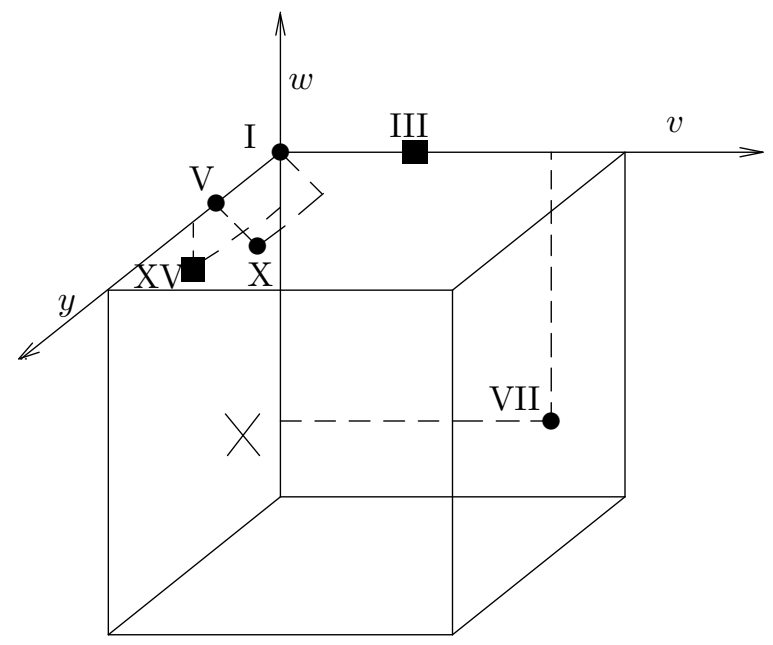

Fig. 12. Fixed points of the RAM with distribution of a local anisotropy axis along hypercube axis for $u=0$. The only fixed points located in the region $v>0, w<0$ are shown. Filled boxes show the stable fixed points, the cross denotes typical initial values of couplings.

The results of the application of the Padé-Borel resummation procedure (45)(47) to the two-loop $\beta$-functions (75)-(78) at $d=3$ are given in Refs. 27.228 where 16 fixed points were obtained for this model. In the Table 9 we present the numerical values of fixed points coordinates with $u^{*}>0, v^{*}>0, w^{*}<0$. The last fixed point XV in Table 9 corresponds to the stable fixed point of $\sqrt{\varepsilon}$-expansion. It has coordinates with $u^{*}=v^{*}=0, w^{*}<0$ and $y^{*}>0$ and is accessible from the typical initial values of couplings (marked by a cross in the Fig. 12). The Padé-Borel resummation procedure (75)-(78) does not reveal any other stable fixed points in the region of interest. Thus one draws the conclusion that the system in the critical regime is characterized by the same set of critical exponents as the weakly diluted quenched Ising model.

In the other fixed points, one recovers the familiar two-loop numerical results for the Gaussian (fixed points I, VII), $m$-vector (II), polymer $O(n=0)$ (III), Ising $(\mathrm{V}, \mathrm{X})$, diluted $m$-vector (VI), and cubic (VIII) models. Fixed point IX belongs to a new universality class. In the Table 9 we give the numerical values of the critical exponents in these fixed points as well: if the flow from the initial values of couplings passes near these fixed points one may observe an effective critical behavior governed by these critical exponents. Note that the fixed point VI has a singularity for $m=1$ 
which formally leads to the $\sqrt{\varepsilon}$ expansion. However, as it follows already from the microscopic Hamiltonian (13) for $m=1$ the RAM degenerates to the regular Ising model. This also may be seen from the effective Hamiltonians (28), (29): in the replica limit at $m=1 v$ and $w$ terms (and for (29) also $u$ and $y$ terms) appear to be of the same symmetry. Furthermore, couplings $v$ and $w$ appear to have opposite signs and the same absolute value, therefore they are canceled and the problem reduces to the scalar $\phi^{4}$ theory with only one coupling 130.

As a possible generalization of the RAM one may consider the case when the quenched randomness is present in both the random-site and the random-anisotropy form. This is described by effective Hamiltonians (28), (29) where the coupling $v_{0}$ may be of either sign. We have checked the region $v<0$ for the presence of new fixed points and verified that they are absent. Therefore, again the fixed point XV is the only reachable and stable one and the observed critical behavior is unique. As an interesting extension of these studies one may consider the influence of other types of disorder in combination with the random anisotropy as well as of other random axis distributions.

Table 9. Resummed values of thents points and critical exponents for cubic distribution in two-loop approximation for $d=3127,128$.

\begin{tabular}{|c|c|c|c|c|c|c|c|}
\hline FP & $m$ & $u^{*}$ & $v^{*}$ & $w^{*}$ & $y^{*}$ & $\nu$ & $\eta$ \\
\hline I & $\forall m$ & 0 & 0 & 0 & 0 & $1 / 2$ & 0 \\
\hline & 2 & 0.9107 & 0 & 0 & 0 & 0.663 & 0.027 \\
II & 3 & 0.8102 & 0 & 0 & 0 & 0.693 & 0.027 \\
& 4 & 0.7275 & 0 & 0 & 0 & 0.720 & 0.026 \\
\hline III & $\forall m$ & 0 & 1.1857 & 0 & 0 & 0.590 & 0.023 \\
\hline V & $\forall m$ & 0 & 0 & 0 & 1.0339 & 0.628 & 0.026 \\
\hline VI & 3 & 0.6460 & 0.1733 & 0 & 0 & 0.659 & 0.027 \\
& 4 & 0.4851 & 0.2867 & 0 & 0 & 0.653 & 0.027 \\
\hline VII & $\forall m$ & 0 & 2.1112 & -2.1112 & 0 & $1 / 2$ & 0 \\
\hline & 2 & 1.5508 & 0 & 0 & -1.0339 & 0.628 & 0.026 \\
VIII & 3 & 0.8393 & 0 & 0 & -0.0485 & 0.693 & 0.027 \\
& 4 & 0.5259 & 0 & 0 & 0.3624 & 0.709 & 0.026 \\
\hline IX & 3 & 0.7096 & 0.1695 & 0 & -0.1022 & 0.659 & 0.027 \\
& 4 & 0.4190 & 0.2751 & 0 & 0.1432 & 0.653 & 0.027 \\
\hline $\mathrm{X}$ & $\forall m$ & 0 & 0.6678 & -0.6678 & 1.0339 & 0.628 & 0.026 \\
\hline $\mathrm{XV}$ & $\forall m$ & 0 & 0 & -0.4401 & 1.5933 & 0.676 & 0.031 \\
\hline
\end{tabular}

As we have seen from the analysis of the subsection 6.3 the local anisotropy axis distribution has a crucial influence on the scenarios of low-temperature behavior. For the RAM with isotropic distribution the inaccessibility of any stable fixed point leads to the conclusion that a second order phase transition is absent. The existence of a reachable disordered Ising-like fixed point in case of the RAM with cubic distribution of the random anisotropy axis indicates that the system undergoes a second order phase transition with the critical exponents of the weakly diluted quenched Ising model. Here, we want to attract the readers attention to the similarity of the critical behavior of both the random-site 76 and the random-anisotropy 18 quenched 
magnets: if new critical behavior is present then it is always governed by the critical exponents of the site-diluted Ising type. The above calculations of a "phase diagram" of the RAM are based on two-loop expansions improved by a resummation technique. Once the qualitative picture becomes clear there is no need to go into higher orders of a perturbation theory as far as the critical exponents of site-diluted Ising model are known by now with high accuracy (see Table 3 and discussion of the subsection 6.1).

\section{Conclusions}

To conclude this review, let us return to the question posed in the introduction: what information about the influence of weak quenched disorder on criticality can one get analyzing the RG expansions? Comparing the results of the theoretical analysis in Section 6 with the available data about different physical phenomena listed in Section 3 one definitely can state that in most cases the RG analysis provides a correct description. Indeed, the change of the universality class of the Ising model upon weak quenched dilution predicted by the $\mathrm{RG}$ analysis is confirmed by numerous experiments on diluted uniaxial magnets $\mathrm{Fe}_{\mathrm{p}} \mathrm{Zn}_{1-\mathrm{p}} \mathrm{F}_{2}, \mathrm{Mn}_{\mathrm{p}} \mathrm{Zn}_{1-\mathrm{p}} \mathrm{F}_{2}$ as well as by extensive MC simulations (c. f. data of the Table 3 and of the Section (3). By high precision experiments under microgravity conditions it was shown 42 that the specific heat asymptotic critical exponent of liquid $\mathrm{He}^{4}$ for the $\lambda$-transition is not influenced by quenched disorder and belongs to the universality class of the $m=2$ Stanley model. This result also follows from the RG analysis as far as the Stanley model heat capacity critical exponent $\alpha$ changes its sign already for $m<2131$. The MC simulations of self-avoiding walks on random-site lattices 65 .66 confirmed the theoretical result of Ref. 62 that universal properties of self-avoiding walks are not influenced by point-like uncorrelated disorder. The change of the universality class of self-avoiding walks by a long-range-correlated disorder predicted by the RG analysis 69 waits for an experimental or simulational confirmation. The MC study of the influence of the long-range-correlated disorder on the ferromagnetic phase transition confirms the change of the universality class in this case 5 . One more interesting check of the $\mathrm{RG}$ predictions might be the verification (experimental or in MC simulations) of the envisaged random Ising model critical behavior for the random anisotropy magnet with a cubic distribution of the anisotropy axis 127, $228,129.132$.

As we have seen in these lectures, for the majority of the phenomena listed above a careful analysis of the RG expansions provides also a quantitative description, giving reliable numbers for the physical values describing criticality. However, if one compares the RG expansions for non-diluted ("pure") and diluted systems one certainly notices essential differences. A typical scenario that one encounters when analyzing the critical properties of the $d=3$ Stanley model (i.e. applying the field theoretical RG technique to the $O(m)$-symmetrical $\phi^{4}$ model) may be sketched by two steps: (i) already in the first non-trivial order of the perturbation theory one 
observes a new non-trivial critical behavior (a stable non-trivial fixed point); (ii) the higher order contributions do not change the picture qualitatively and improve the numerical outcome provided the resummation is applied. This scenario does not hold for most of the models with disorder. Here, the first-order perturbation theory expansions as a rule do not lead to new criticality (a non-trivial fixed point is absent or its stability is questioned). The (experimentally confirmed!) answer about new critical behavior is obtained only by considering higher order contributions with the help of the resummation technique. But then again, even the resummed series appear to be divergent starting from some order of perturbation theory showing an "optimal truncation" behavior. Some expansions that are familiar and useful for the "pure" models appear to be of no use for certain disordered models: e.g. the $\varepsilon$-expansion turns into the $\sqrt{\varepsilon}$-expansion for the random-site Ising model and does not lead to any quantitative information.

Technically, this "strange" behavior of the perturbation theory expansions for the disordered models is not strange: it rather signals the divergent nature of the series. For some disordered models this behavior was shown 44.85 to originate from Griffith-like singularities 83 caused by zeroes of the partition function of the pure system. The physical reason for Griffith singularities is the existence of ordered islands within the temperature interval below the critical temperature of the pure model and above that of the diluted model. So in this sense the studies of random models reviewed above may serve as examples when more complicated physics leads to a more complicated theoretical description. And as one may conclude from the results obtained so far in many cases this description is a fruitful one.

\section{Acknowledgments}

The materials presented here were the subject of lectures given by one of us in summer semester 2001 in the Université Henri Poincaré, Nancy 1 (France) and at the 2nd Pamporovo workshop on cooperative phenomena in condensed matter (August 2001, Bulgaria). Yu.H. expresses his thanks to Bertrand Berche and colleagues at the Laboratoire de physique des matériaux for their hospitality, support and many useful comments. It is also a special pleasure to express our gratitude to Dimo I. Uzunov and his colleagues from the G. Nadjakov Institute of Solid State Physics of the Bulgarian Acad. Sci. for their devoted work on organization of Pamporovo workshops.

M. D. acknowledges the Ernst Mach research fellowship of the Österreichisher Austauschdienst. This work was supported in part by Österreichische Nationalbank Jubiläumsfonds through grant No 7694 .

\section{References}

1. G. Forgacs, R. Lipowsky, Th. Nieuwenhuizen, in Phase Transitions and Critical Phenomena, ed. by C. Domb and J. L. Lebowitz, (Academic Press, London, 1991), vol. 14 . 
2. E. Brézin, J. C. Le Guillou, J. Zinn-Justin, in Phase Transitions and Critical Phenomena, ed. by C. Domb and M. S. Green, (Academic Press, London, 1976), vol. 6; D. J. Amit, Field Theory, the Renormalization Group, and Critical Phenomena, (World Scientific, Singapore, 1989); J. Zinn-Justin, Quantum Field Theory and Critical Phenomena, (Oxford University Press, 1996); H. Kleinert, V. Schulte-Frohlinde, Critical Properties of $\phi^{4}$-Theories, (World Scientific, Singapore, 2001).

3. H. E. Stanley, Phys. Rev. Lett 20, 589 (1968)

4. Absence of the phase transition with spontaneous order parameter for the systems with continuous symmetry and a short range interaction at $d=2$ follows from the Mermin-Wagner theorem: N. D. Mermin and N. Wagner, Phys. Rev. Lett. 17, 1133 (1966); exact solutions for $2 \mathrm{~d}$ Stanley model for $m=4$ and $m=3$ also demonstrate the absence of magnetic ordering: A. M. Polyakov and P. B. Wiegmann, Phys. Lett. B131, 121 (1983); P. B. Wiegmann, Pis'ma Zh. Eksp. Teor. Fiz. 41, 79 (1985) [JETP Letters 41, 95 (1985)].

5. See e.g. H. E. Stanley, Introduction to Phase Transitions and Critical Phenomena, (Claredon Press, Oxford 1971).

6. R. Guida, J. Zinn-Justin J. Phys. A 31, 8103 (1998).

7. One can account the presence of non-magnetic impurities introducing random interaction $J$. However, the corresponding random-bond model is conjectured to exhibit universal critical behavior identical to the site-diluted model: K. Hukushima J. Phys. Soc. Jap. 69, 631 (2000); P. E. Berche, C. Chatelain, B. Berche, W. Janke, in MECO25, Middle European Cooperation in Statistical Physics, (Pont-à-Mousson, France, 2000), p.51.

8. See e.g. J. W. Essam, in Phase Transitions and Critical Phenomena, ed. by C. Domb and M. S. Green, (Academic Press, London, 1972), vol. 2.

9. R. Brout, Phys. Rev. 115, 824 (1959).

10. M. E. Fisher, Phys. Rev. 176, 257 (1968).

11. A. B. Harris J. Phys. C 7, 1671 (1974).

12. One should be careful in applying the Harris criterion "naively" to the SAW problem: see discussion in the subsection 3.6.

13. J. T. Chayes, L. Chayes, D. S. Fisher, T. Spenser Phys. Rev. Lett. 57, 2999 (1986).

14. A. Weinrib and B.I. Halperin, Phys. Rev. B27, 413 (1983).

15. S. M. Dorogovtsev, Fiz. Tverd. Tela (Leningrad) 22:321 (1980) [Sov. Phys.-Solid State22:188 (1980)]

16. A. L. Korzhenevsky, K. Herrmanns, W. Schirmacher, Phys. Rev. B53:14834 (1996).

17. Y. Yamazaki, A. Holz, M. Ochiai, and Y. Fukuda, Physica A 150, 576 (1988).

18. R. Harris, M. Plischke, and M. J. Zuckermann, Phys. Rev. Lett. 31, 160 (1973).

19. A. Aharony, Phys. Rev. B12, 1038 (1975).

20. Y. Imry, S.-k. Ma, Phys. Rev. Lett. 35, 1399 (1975).

21. See e. g. C. Jayaprakash and S. Kirkpatrick, Phys. Rev. B21, 4072 (1980) and Y. Y. Goldschmidt and A. Aharony, Phys. Rev. B32, 264 (1985).

22. R. A. Pelcovits, E. Pytte, and J. Rudnick, Phys. Rev. Lett. 40, 476 (1978).

23. R. A. Dunlap and A. M. Gottlieb, Phys. Rev. B23, 6106 (1981).

24. R. J. Birgeneau, R. A. Cowley, G. Shirane, H. Joshizawa, D. P. Belanger, A. R. King, and V. Jaccarino, Phys. Rev. B27, 6747 (1983).

25. D. P. Belanger, A. R. King, and V. Jaccarino, Phys. Rev. B 34, 452 (1986).

26. P. W. Mitchell, R. A. Cowley, H. Yoshizawa, P. Böni, Y. J. Uemura, and R. J. Birgeneau, Phys. Rev. B34, 4719 (1986).

27. P. H. Barrett, Phys. Rev. B34, 3513 (1986).

28. T. R. Thurston, C. J. Peters, R. J. Birgeneau, and P. M. Horn, Phys. Rev. B37, 

9559 (1988).

29. N. Rosov, A. Kleinhammes, P. Lidbjörk, C. Hohenemser, and M. Eibschütz, Phys. Rev. B37, 3265 (1988).

30. S. Fishman and A. Aharony, J. Phys. C 12, L729 (1979); J. Cardy, Phys. Rev. B29, 505 (1984).

31. C. A. Ramos, A. R. King, and V. Jaccarino, Phys. Rev. B37, 5483 (1988); I. B. Ferreira, A. R. King, and V. Jaccarino, Phys. Rev. B43, 10797 (1991); D. P. Belanger, J. Wang, Z. Slanic, S.-J. Han, R. M. Nicklow, M. Lui, C. A. Ramos, and D. Lederman, Journ. Magn. Magn. Mater. 140, 1549 (1995), Phys. Rev. B54, 3420 (1996); J. P. Hill, Q. Feng, Q. J. Harris, R. J. Birgeneau, A. P. Ramirez, and A. Cassanho, Phys. Rev. B55, 356 (1997); Z. Slanič, D. P. Belanger, and J. A. Fernandez-Baca, J. Magn. Magn. Mater. 177, 171 (1998); Z. Slanič and D. P. Belanger, J. Magn. Magn. Mater. 186, 65 (1998), Phys. Rev. Lett. 82, 426 (1999).

32. R. Folk, Yu. Holovatch, T. Yavors'kii, cond-mat/0106468 (2001), to appear in Physics Uspiekhi (2002).

33. H. Yamamoto, H. Onodera, K. Hosoyama, T. Masumoto, and H. Yamauchi, J. Magn. Magn. Mater. 31-34, 1579 (1983); K. Winschuh and M. Rosenberg, J. Appl. Phys. 61, 4401 (1987); G. K. Nicolaides, G. C. Hadjipanayis, and K. V. Rao, Phys. Rev. B 48, 12759 (1993); P. D. Babu and S. N. Kaul, J. Phys.: Cond. Matt. 9, 7189 (1997).

34. A. Perumal, V. Srinivas, K. S. Kim, S. C. Yu, V. V. Rao, and R. A. Dunlap, J. Magn. Magn. Mater. 233, 280 (2001).

35. M. Fähnle, G Herzer, H. Kronmüller, R. Meyer, M. Saile, and T. Egami, J. Magn. Magn. Mater. 38, 240 (1983); S. N. Kaul, Phys. Rev. B 38, 9178 (1988); S. N. Kaul and Ch. V. Mohan, Phys. Rev. B 50, 6157 (1994).

36. W.-U. Kellner, T. Albrecht, M. Fähnle, and H. Kronmüller, J. Magn. Magn. Mater. 62, 169 (1986).

37. O. Boxberg and K. Westerholt, Phys. Rev. B 50, 9331 (1994).

38. K. Westerholt and G. Sobotta, J. Phys. F 13, 2371 (1983); K. Westerholt, H. Bach, and R. Römer, J. Magn. Magn. Mater. 45, 252 (1984); K. Westerholt, Physica 130B, 533 (1985); K. Westerholt, J. Magn. Magn. Mater. 66, 253 (1987).

39. M. Fähnle, J. Magn. Magn. Mater., 45, 279 (1984).

40. M. Dudka, R. Folk, Yu. Holovatch, and D. Ivaneiko, J. Magn. Magn. Mater., (2002), to appear.

41. Only in a special case liquids in porous media are an example for the site diluted Ising model; otherwise they are conjectured to be examples for the random-field models [ see e.g. E. Pitard, M. L. Rosinberg, G. Stell, G. Tarjus, Phys. Rev. Lett. 74, 4361 (1995)]

42. J. Yoon, M. H. W. Chan, Phys. Rev. Lett. 78, 4801 (1997); see also: G. M. Zassenhaus, J. D. Reppy Phys. Rev. Lett. 83, 4800 (1999).

43. Experimentally measured value of the heat capacity critical exponent at the $\lambda$ transition in helium- 4 reads: $\alpha=-0.01056 \pm 0.00038$ [J. A. Lipa, D. R. Swanson, J. A. Nissen, Z. K. Geng, P. R. Williamson, D. A. Stricker, T. C. P. Chui, U. E. Israelsson, M. Larson, Phys. Rev. Lett. 84, 4894 (1999)]. Within the error bars $\alpha<0$ therefore due to the Harris criterion a weak quenched disorder should not influence the critical exponents.

44. A. Pelissetto and E. Vicari, cond-mat/0012164 (2000), to appear in Physics Reports (2002).

45. D. P. Landau, Phys. Rev. B 22, 2450 (1980).

46. J. Marro, A. Labarta, and J. Tejada, Phys. Rev. B34, 347 (1986); D. Chowdhury and D. Staufer, J. Stat. Phys. 44, 203 (1986). 
47. J.-S. Wang and D. Chowdhury, J. Phys. France 50, 2905 (1989).

48. J.-S. Wang, M. Wöhlert, H. Mühlenbein, and D. Chowdhury, Physica A 166, 173 (1990).

49. H.-O. Heuer, Europhys. Lett. 12, 551 (1990); H.-O. Heuer, Phys. Rev. B42, 6476 (1990).

50. H.-O. Heuer, J. Phys. A 26, L333 (1993).

51. H. G. Ballesteros, L. A. Fernández, V. Martín-Mayor, A. Muñoz Sudupe, G. Parisi, and J. J. Ruiz-Lorenzo, Phys. Rev. B58, 2740 (1998).

52. R. Folk, Yu. Holovatch and T. Yavorskii, Phys. Rev. B61, 15114 (2000).

53. Y. Yamazaki, A. Holz, M. Ochiai, Y. Fukuda, Phys. Rev. B33, 3460 (1985); Y. Yamazaki, Y. Fukuda, A. Holz, M. Ochiai, Physica A 136, 303 (1986).

54. D. Boyanovsky and J. L. Cardy, Phys. Rev. B26, 154 (1982).

55. H. G. Ballesteros and G. Parisi, Phys. Rev. B60, 12912 (1999).

56. M. Marqués, J. Gonzalo and J. Íniquez, Phys. Rev. E62, 191 (2000).

57. J. C. Lee, R. Gibbs, Phys. Rev. B45:2217 (1992).

58. J. des Cloizeaux, G. Jannink, Polymers in Solution, (Clarendon Press, Oxford, 1990); L. Schäfer, Excluded Volume Effects in Polymer Solutions (Springer, Berlin, 1999).

59. P.-G. de Gennes, Scaling Concepts in Polymer Physics, (Cornell University Press, Ithaca and London, 1979).

60. B. K. Chakrabarti and J. Kertész, Z. Phys. B:Condensed Matter 44, 221 (1981).

61. A. B. Harris, Z. Phys. B 49, 347 (1983).

62. Y. Kim, J. Phys. C 16, 1345 (1983).

63. Y. Meir and A. B. Harris, Phys. Rev. Lett. 63, 2819 (1989).

64. P. Grassberger, J. Phys. A 26, 1023 (1993).

65. K. Barat and B. K. Chakrabarti, Phys. Reports 258, 377 (1995).

66. S. Lee and H. Nakanishi, Phys. Rev. Lett. 61, 2022 (1987); S. Lee, H. Nakanishi and Y. Kim, Phys. Rev. B39, 9561 (1988); J. Machta and T.R. Kirkpatrick, Phys. Rev. A41, 5345 (1990); A.V. Izyumov and K. B. Samokhin, J. Phys. A 32, 7843 (1999); A. R. Altenberger, I. I. Siepmann, and J. S. Dahler, J. Phys. A 272, 22 (1999); Y. Shiferaw and Y. Y. Goldschmidt, J. Phys. A 33:4461 (2000).

67. For recent references see e.g. A. Ordemann, M. Porto, H. Eduardo Roman, S. Havlin, and A. Bunde, Phys. Rev. E61, 6858 (2000); A. Ordemann, M. Porto, H. Eduardo Roman, and S. Havlin, Phys. Rev. E63, 020104(R) (2001).

68. K. Kremer, Z. Phys. B 45, 149 (1981); S. Lee, and N. Nakanishi, Phys. Rev. Lett. 61, 2022 (1988).

69. V. Blavats'ka, C. von Ferber, and Yu. Holovatch, Phys. Rev E64, 041102 (2001).

70. V. Blavats'ka, C. von Ferber, and Yu. Holovatch, J. Mol. Liq. 91, 77 (2001).

71. R. W. Cochrane, R. Harris, and M. J. Zuckermann, Phys. Rep. 48, 1 (1978).

72. R. W. Cochrane, R. Harris, and M. Plischke, J. Non.-Cryst. Solids 15, 239 (1974).

73. R. W. Cochrane, R. Harris, M. Plischke, D. Zobin, and M. J. Zuckermann, J. Phys. F 5, 763 (1975).

74. J. Chakrabati, Phys. Rev. Lett 81, 385 (1998).

75. X.-l. Wu, W. I. Goldburg, M. X. Liu, and J. Z. Xue Phys. Rev. Lett. 69, 470 (1992).

76. G. Grinstein, A. Luther, Phys. Rev. B13, 1329 (1976)

77. V. J. Emery, Phys. Rev. B11, 239 (1975); S. F. Edwards, P. W. Anderson J. Phys. $F$ 5, 965 (1975).

78. One more extension of this model of a random magnet is to take into account both influence of a single-ion anisotropy and random impurities as is done e.g. in I. D. Lawrie, Y. T. Millev, and D. I. Uzunov J. Phys. A 20, 1599 (1987); Erratum: J. Phys. A 20, 6159 (1987). 
79. G. Parisi, in Proceedings of the Cargrése Summer School (1973), unpublished; G. Parisi, J. Stat. Phys. 23, 49 (1980).

80. V.V. Prudnikov, P.V. Prudnikov, and A.A. Fedorenko, J. Phys. A 32, L399 (1999); J. Phys. A 32, 8587 (1999); Phys. Rev. B62, 8777 (2000).

81. G. H. Hardy Divergent Series (Oxford, 1948).

82. J.-P. Eckmann, J. Magnen and R. Sénéor, Commun. Math. Phys. 39, 251 (1975); J. S. Feldman and K. Osterwalder, Ann. Phys. 97, 80 (1976); J. Magnen and R. Sénéor, Commun. Math. Phys. 56, 237 (1977); J.-P. Eckmann and H. Epstein, Commun. Math. Phys. 68, 245 (1979).

83. R. B. Griffiths, Phys. Rev. Lett. 23, 17 (1969)

84. A. J. Bray, T. McCarthy, M. A. Moore, J. D. Reger, and A. P. Young, Phys. Rev. B36, 2212 (1987); A. J. McKane, Phys. Rev. B49, 12003 (1994).

85. G. Álvarez, V. Martín-Mayor, and J. J. Ruiz-Lorenzo, J. Phys. A 33, 841 (2000).

86. L. N. Lipatov, Zh. Eksp. Teor. Fiz. 72, 411 (1977) [Sov. Phys. JETP 45, 216].

87. E. Brézin, J. Le Guillou, and J. Zinn-Justin, Phys. Rev. D15, 1544 (1977).

88. E. Brézin and G. Parisi, J. Stat. Phys. 19, 269 (1978).

89. Note that for finite number of terms changing of order of integration and summation can always be performed

90. G. A. Baker, B. G. Nickel, M. S. Green, and D. I. Meiron, Phys. Rev. Lett. 36, 1351 (1976); G. A. Baker, B. G. Nickel, and D. I. Meiron, Phys. Rev. B17, 1365 (1978).

91. The construction of the Borel-image for the functions of more than two variables is performed similarly to the procedure of the Eq.(48).

92. P. J. S. Watson, J. Phys. A 7, L167 (1974).

93. J. S. R. Chisholm, Math. Comp. 27, 841 (1973); G. A. Baker Jn., P. Graves-Morris, Padé Approximants, (Addison-Wesley, Reading, MA, 1981).

94. A. I. Sokolov, Fiz. Tv. Tela 19, 748 (1977) [Sov. Phys.: Solid State 19, 433].

95. A. I. Sokolov and B. N. Shalaev, Fiz. Tv. Tela 23, 2058 (1981) [Sov. Phys.: Solid State 23, 1200].

96. M. A. Shpot, Phys. Lett. A 142, 474 (1989).

97. I. O. Mayer, A. I. Sokolov, and B. N. Shalaev, Ferroelectrics 95, 93 (1989).

98. D. V. Pakhnin and A. I. Sokolov, Pis'ma v ZhETF 71, 600 (2000). [JETP Letters 71, 412]; D. V. Pakhnin and A. I. Sokolov, Phys. Rev. B61, 15130 (2000).

99. J. M. Carmona, A. Pelissetto, and E. Vicari, Phys. Rev. B61, 15136 (2000).

100. Yu. Holovatch and M. Shpot, J. Stat. Phys. 66, 867 (1992).

101. Values of the loop integrals for fixed space dimension $d=2, d=3$ are given in: B. G. Nickel, D. J. Meiron, and G. A. Baker, "Compilation of 2pt and 4pt graphs for continuous spin model", University of Guelph Report (1977).

102. K. G. Wilson and M. E. Fisher, Phys. Rev. Lett. 28, 240 (1972).

103. G. 't Hooft and M. Veltman, Nucl. Phys. B 44, 189 (1972); G. 't Hooft, Nucl. Phys. B 61, 455 (1973).

104. H. K. Janssen, K. Oerding, and E. Sengespeick, J. Phys. A 28, 6073 (1995).

105. H. Kleinert and V. Schulte-Frohlinde, Phys. Lett. B 342, 284 (1995).

106. D. E. Khmel'nitskii, Zh. Eksp. Teor. Fiz. 68, 1960 (1975) [Sov. Phys. JETP 41, 981]; A. B. Harris and T. C. Lubensky, Phys. Rev. Lett. 33, 1540 (1974); T. C. Lubensky, Phys. Rev. B11, 3573 (1975).

107. B. N. Shalaev, S. A. Antonenko, and A. I. Sokolov, Phys. Lett. A 230, 105 (1997).

108. R. Folk, Yu. Holovatch, and T. Yavors'kii, Pis'ma v ZhETF 69, 698 (1999) [JETP Letters 69, 747]

109. B. N. Shalaev, Zh. Eksp. Teor. Fiz. 73, 2301 (1977) [Sov. Phys. JETP 46, 1204].

110. C. Jayaprakash and H. J. Katz, Phys. Rev. B16, 3987 (1977). 
111. R. Folk, Yu. Holovatch, and T. Yavors'kii, J. Phys. Stud. 2, 213 (1998).

112. J. Jug, Phys. Rev. B27, 609 (1983).

113. I. O. Mayer and A. I. Sokolov, Fiz. Tv. Tela 26, 3454 (1984) [Sov. Phys.: Solid State 26, 2076]

114. I. O. Mayer, J. Phys. A 22, 2815 (1989).

115. K. B. Varnashev, Phys. Rev. B61, 14660 (2000).

116. Yu. Holovatch and T. Yavors'kii, J. Stat. Phys., 93, 785 (1998); Cond. Mat. Phys. iss.11, 87 (1997).

117. R. Folk, Yu. Holovatch, and T. Yavors'kii, unpublished.

118. A. Pelissetto and E. Vicari, Phys. Rev. B62, 6393 (2000).

119. Error bars of the numerical data obtained by an analysis of the perturbation theory series result from comparison of successive orders relative contributions. That is why if only several initial orders of the expansion are available the authors usually do not provide error bars in explicit form discussing rather the confidence interval (see the upper part of the Table 3 as well as tables 月, 7, 9).

120. V. Blavats'ka and Yu. Holovatch, J. Phys. Stud., 5, 261 (2001).

121. K. E. Newman and E. K. Riedel, Phys. Rev. B25, 264 (1982).

122. M. T. Tissier, D. Mouhanna, J. Vidal, and B. Delamotte, cond-mat/0109176 (2001).

123. V. Blavats'ka, C. von Ferber, and Yu. Holovatch, Acta Physica Slovaca (2002), to appear.

124. M. Dudka, R. Folk, and Yu. Holetch, Condes. Matter Phys. 4, 77 (2001).

125. In order to recover result of Ref. 19 one must extract value $\sim 1 / \varepsilon$ of one-loop integral from conventionally normalized couplings.

126. The order $\varepsilon$-expansion contributions to the fixed point values reported in Refs. 124128 contain some errors. This also causes the devarder $5 \%$ of the fixed point VIII numerical value for isotropic distribution 124, 127. Here, we give the corrected numbers.

127. M. Dudka, R. Folk, and Yu. Holovatch, in Fluctuating Path and Fields ed. by W. Janke, A. Pelster, H.-J. Schmidt and M. Bachmann, ( World Scientific, Singapore, 2001), p.457.

128. M. Dudka, R. Folk, and Yu. Holovatch, Condens. Matter Phys. 4, 459 (2001).

129. D. Mukamel and G. Grinstein, Phys. Rev. B25, 381 (1982).

130. The same reasoning holds also for a formal singularity at $m=1$ of the fixed point IV of the RAM with isotropic distribution of the random anisotropy axis.

131. C. Bervillier, Phys. Rev. B34, 8141 (1986); M. Dudka, Yu. Holovatch, and T. Yavors'kii, J. Phys. Stud. 5, 233 (2001).

132. A. L. Korzhenevskii and A. A. Luzhkov, Zh. Eksp. Teor. Fiz. 94, 250 (1988) [Sov. Phys. JETP 67, 1229]. 